\title{
Meroterpenoid Synthesis via Sequential Polyketide Aromatization and Cationic Polyene Cyclization: Total Syntheses of $(+)$-Hongoquercin A and B and Related Meroterpenoids
}

\author{
Tsz-Kan Ma, ${ }^{\dagger, \ddagger}$ Daniel C. Elliott, ${ }^{\dagger, \ddagger}$ Stephanie Reid, ${ }^{\dagger, \ddagger}$ Andrew J. P. White, ${ }^{\dagger, \ddagger}$ Philip J. Parsons, ${ }^{*}, \dagger, \ddagger$
} and Anthony G. M. Barrett*, ${ }^{*},+\infty$

${ }^{\dagger}$ Department of Chemistry, Imperial College, London, SW7 2AZ, England

${ }^{\ddagger}$ Department of Chemistry, Imperial College London, Molecular Sciences Research Hub, White City Campus, Wood Lane,London W12 0BZ, England

Supporting Information

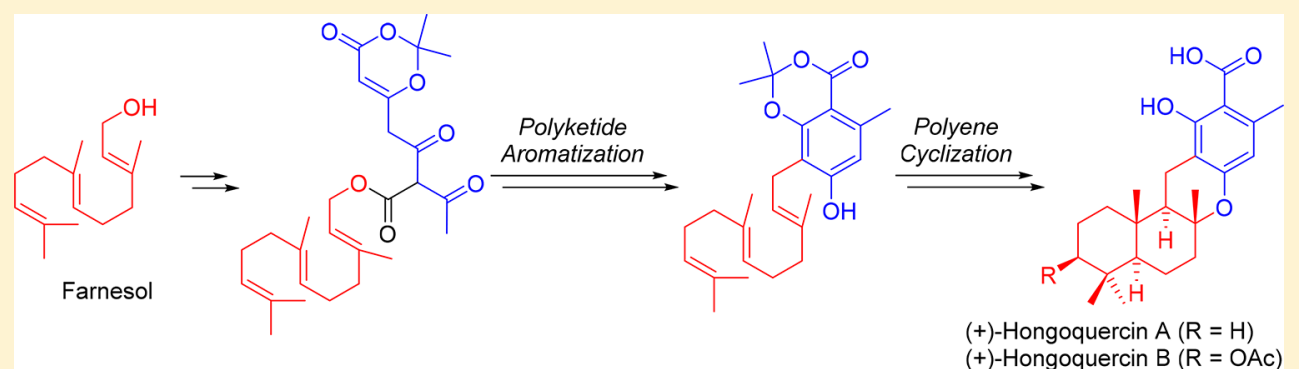

ABSTRACT: (+)-Hongoquercin A and B were synthesized from commercially available trans,trans-farnesol in six and eleven steps, respectively, using dual biomimetic strategies with polyketide aromatization and subsequent polyene functionalization from a common farnesyl-resorcylate intermediate. Key steps involve $\mathrm{Pd}(0)$-catalyzed decarboxylative allylic rearrangement of a dioxinone $\beta, \delta$-diketo ester to a $\beta, \delta$-diketo dioxinone, which was readily aromatized into the corresponding resorcylate, and subsequent polyene cyclization via enantioselective protonation or regioselective terminal alkene oxidation and cationic cyclization of enantiomerically enriched epoxide to furnish the tetracyclic natural product cores. Analogues of the hongoquercin were synthesized via halonium-induced polyene cyclizations, and the meroterpenoid could be further functionalized via saponification, hydrolytic decarboxylation, reduction, and amidation reactions.

\section{INTRODUCTION}

(+)-Hongoquercin A (1) and B (2) were isolated from the fermentation broths produced by an unidentified fungus in 1998 independently by Roll and Abbanat (Figure 1). ${ }^{1}$ They exhibited modest antibacterial activity against methicillinresistant Staphylococcus aureus and vancomycin-resistant Enterococcus faecium. ${ }^{1}$ These natural products are meroterpenoids which have a mixed biosynthetic origin involving polyketide and terpenoid pathways. (-)-Siccanin (3) and (-)-austalide $\mathrm{K}$

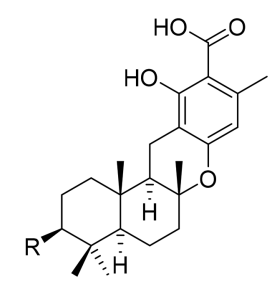

(+)-Hongoquercin $\mathrm{A}(1)(\mathrm{R}=\mathrm{H})$ $(+)$-Hongoquercin $B(2)(R=O A c)$
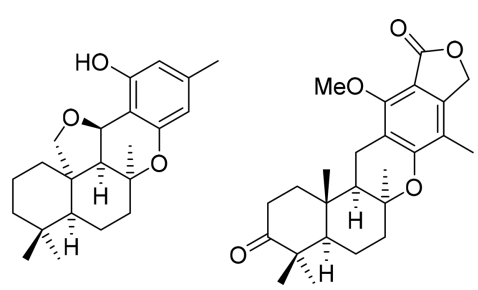

$(-)-S i c c a n i n(3)$

(-)-Austalide K (4)
(4) are additional examples of such structurally diverse bioactive meroterpenoids. ${ }^{2}$

Over the last two decades, several total syntheses of hongoquercins 5 have been reported. ${ }^{3}$ The common synthetic strategy involves the coupling of two synthons, an enantiopure synthesized drimene $\mathbf{6}$ coupled with a substituted resorcinol derivative 7 (Scheme 1). However, this conventional approach often requires extensive use of protecting groups on the resorcinol unit 7 and multistep transformations for the synthesis of the precursor drimene 6. Most of the reported processes for the preparation of the resorcinol rely on extensive derivatization of an aromatic precursor, while alternative synthetic strategies to prepare resorcinol, such as benzannulation, have been shown to be more concise and flexible. ${ }^{4}$ Therefore, we considered that a dual biomimetic approach for elaborating the arene ring and tricyclic terpenoid residues from acyclic precursor 9 sequentially via cascade cyclizations would simplify the syntheses of these natural products. Additionally, if the farnesyl residue was functionalized after aromatization to

Received: August 13, 2018

Published: October 15, 2018 
Scheme 1. Synthetic Strategies of the Hongoquercins 5 Conventional Approach<smiles>[R]C1CC[C@]2(C)[C@@H](CC[C@@]3(C)Oc4cc(C)c(C(=O)O)c(O)c4C[C@@H]32)C1(C)C</smiles><smiles>[X]CC1=C(C)CC[C@H]2C(C)(C)C([R])CC[C@]12C</smiles><smiles>[R6]OC(=O)c1c(C)cc(O[R6])c([Y])c1O[R6]</smiles>

Dual Biomimetic Approach (This work)

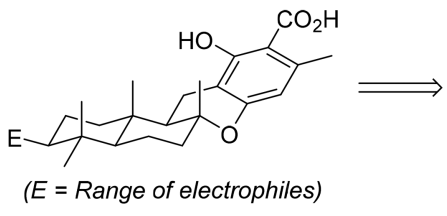

8

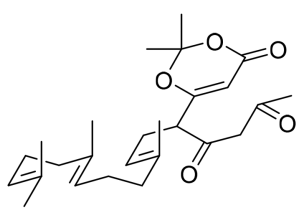

9

construct the resorcylate entity, a diverse range of hongoquercin analogues 8 should be available by variation of the electrophilic reagents used in such derivatizations.

Inspired by the pioneering work of the Harris group and Hyatt group, respectively, on the biomimetic synthesis of $\beta$ resorylate and on the generation of acyl ketenes by the thermolysis of dioxinones, ${ }^{5,6}$ our group has developed a biomimetic route to $\beta$-resorylate natural products that utilizes $\beta, \delta$-diketo dioxinones as masked triketo ketenes. ${ }^{7}$ In 2009 , we additionally discovered a regioselective palladium $(0)$-catalyzed decarboxylative rearrangement during the synthesis of aigialomycin D. ${ }^{8}$ Application of this reaction greatly facilitated the synthesis of meroterpenoid resorcylate natural products. ${ }^{9}$ More recently, we developed an efficient methodology for the synthesis of dioxinone $\beta$-keto esters 12 using dioxane-4,6-dione keto dioxanones $\mathbf{1 0}$ as the masked dioxinone acylketene $\mathbf{1 1}$ (Scheme 2). ${ }^{10}$ Application of this reaction provided an efficient

\section{Scheme 2. Thermolysis of Dioxane-4,6-dione}

Ketodioxanones 10<smiles>[R]C1([Tl])OC(=O)C(=C(O)CC2=CC(=O)OC(C)(C)O2)C(=O)O1</smiles>

$(\mathrm{R}=\mathrm{H}$ or $\mathrm{Me})$

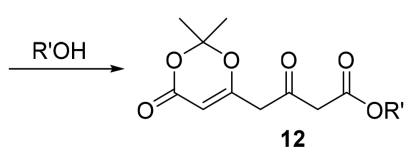

route for the synthesis of $\beta$-resorylates, and its utility has been showcased in the total syntheses of several bioactive meroterpenoid natural products. ${ }^{10}$ Herein we report further extensive studies on the dual biomimetic total synthesis of the hongoquercins $\mathbf{5}$, which we initially published as a communication. ${ }^{9 \mathrm{e}}$

\section{RESULTS AND DISCUSSION}

We considered that the key meroterpenoids 13 should be available using a polyene cyclization from resorcylate 14 by enantioselective electrophilic reactions with chiral Brønsted acids $(E=H)$, epoxidation, and subsequent reaction with a

Lewis acid $(\mathrm{E}=\mathrm{OH})$ or halogenations with reagents that provide halonium ion intermediates $(\mathrm{E}=\mathrm{Br}$ and $\mathrm{I}$ ) (Scheme 3). The common resorcylate intermediate $\mathbf{1 4}$ should be

Scheme 3. Retrosynthetic Analyses of Hongoquercins A (1) and $B(2)$

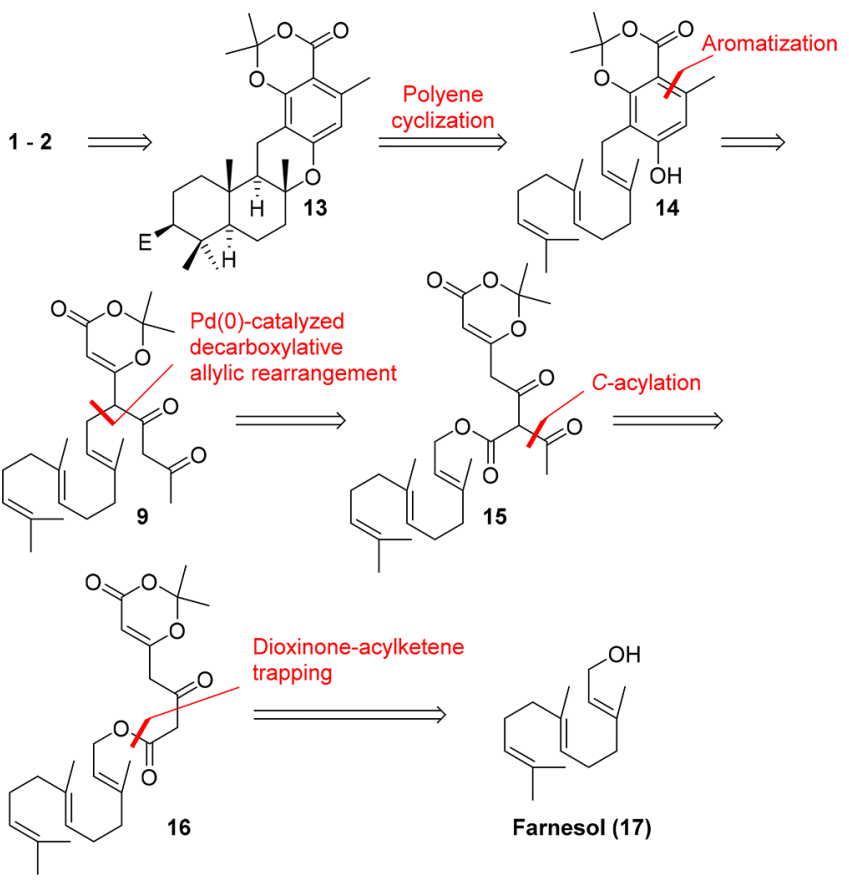

available from the cycloaromatization of $\beta, \delta$-diketodioxinone 9 , which could be synthesized via palladium $(0)$-catalyzed decarboxylative allylic rearrangement of dioxinone $\beta, \delta$-diketo ester 15. Dioxinone $\beta, \delta$-diketo ester 15 , in turn, should be available via $C$-acylation of dioxinone $\beta$-keto ester 16 , which, in turn, is available from trapping a dioxinone acylketene with trans,trans-farnesol $(\mathbf{1 7}){ }^{10}$

Following our recently published methods, ${ }^{10}$ thermolysis of dioxane-4,6-dione keto dioxanone 19 at $55{ }^{\circ} \mathrm{C}$ generated the dioxinone acyl ketene 11, which was trapped with trans,transfarnesol (17) to provide dioxinone $\beta$-keto ester 21 (79\%) (Scheme 4). Magnesium chloride mediated regioselective $C$ acylation of $\beta$-keto ester $\mathbf{2 1}$ with acetyl chloride gave dioxinone $\beta, \delta$-diketo ester 23 , which on reaction with $\mathrm{Pd}_{2}(\mathrm{dba})_{3}$ and tri(2-furyl)phosphine resulted in a highly regioselective decarboxylative allylic rearrangement giving the $\beta, \delta$-diketo dioxinone 9 and readily aromatized in situ to produce farnesyl resorcylate 14 (55\% overall from 21). A geranyl-substituted analogue 24 was also synthesized, using the same reaction sequence, from geraniol (18) in three steps with an overall yield of $57 \%$.

We first investigated the synthesis of (+)-hongoquercin A (1) via enantioselective protonation of farnesyl resorcylate 14 (Scheme 5) using the Lewis acid enhanced chiral Brønsted acids derived from antimony pentachloride with binol 25 and stannic chloride with binol $\mathbf{2 6}$ as introduced by Corey et al. ${ }^{11}$ and Yamamoto et al. ${ }^{12}$ Enantioselective protonation with $\mathrm{SbCl}_{5} \cdot 25$ gave a mixture of partially cyclized products from which the desired meroterpenoid 23 was isolated in $15 \%$ yield and with an enantiomeric excess of $20 \%$ as determined by chiral HPLC. Fortunately, the cyclization using $\mathrm{SnCl}_{4} \cdot 26$ as the dual Brønsted and Lewis acids was highly enantioselective and 
Scheme 4. Synthesis of the Terpene Resorcylates 14 and 24

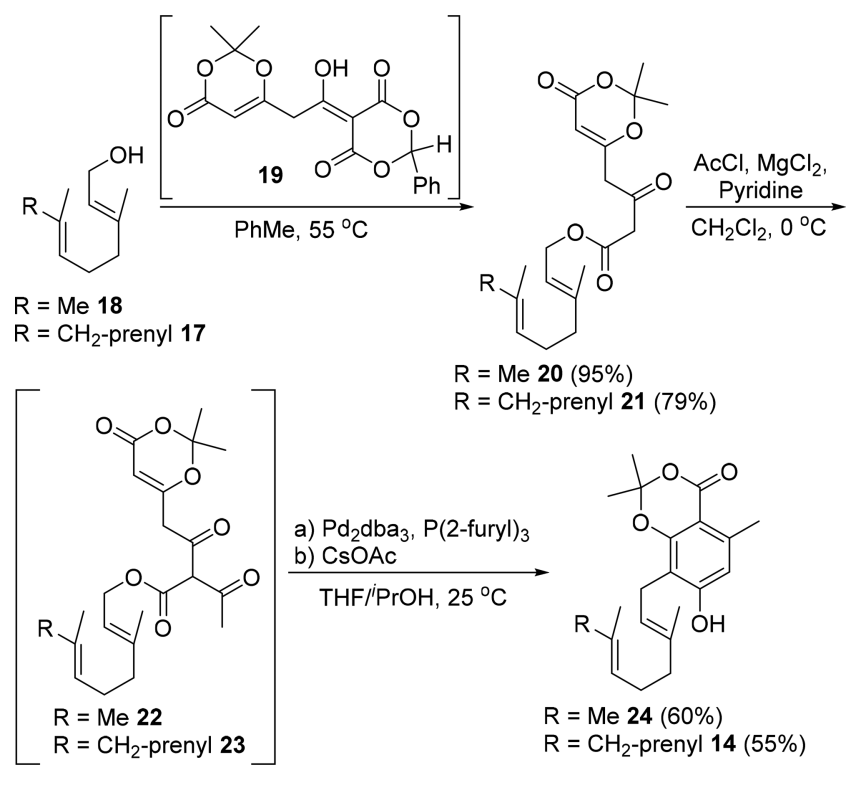

Scheme 5. Total Synthesis of (+)-Hongoquercin A (1)
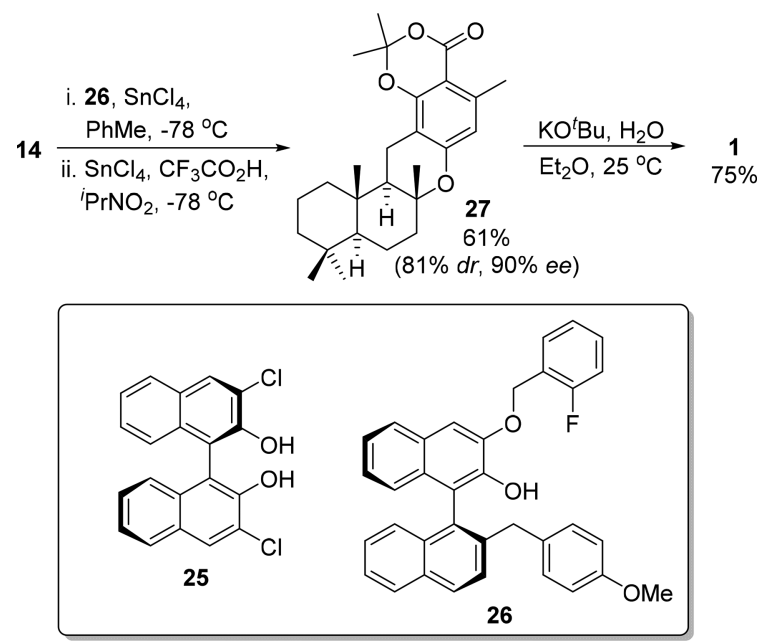

gave the desired meroterpenoid $27(61 \%, 81 \% \mathrm{dr}$ and $90 \%$ ee as determined by chiral HPLC) on sequential reaction with $\mathrm{SnCl}_{4} \cdot 26$ and $\mathrm{SnCl}_{4}$ and trifluoroacetic acid. Finally, saponification ${ }^{13}$ of meroterpenoid 27 gave (+)-hongoquercin A (1) (75\%) with an overall yield of $20 \%$ over five 5 steps from trans,trans-farnesol 17. The spectroscopic data were in full agreement with that reported for the isolated natural product, ${ }^{1}$ and the structure was unambiguously confirmed by singlecrystal X-ray crystallography.

Next, we focused on the synthesis of (+)-hongoquercin B, which utilized enantioselective epoxidation (Scheme 6). While we had reported the synthesis of this natural product from the farnesyl derivative $33,{ }^{9 \mathrm{e}}$ we wished to reinvestigate this synthesis with late-stage oxidation of the terminal alkene on the pendant farnesyl side chain since this should greatly facilitate the synthesis and bioassay of a focused library of novel hongoquercin analogues with the late-stage introduction of terpene structural diversity. In order to effect such electrophilic functionalization of the terminal alkene unit, we needed to suppress phenol-directed oxidation. ${ }^{14}$ We found that protection by phenol allylation was suitable for this purpose.
Allylation of farnesyl resorcylate 14 gave allyl ether 28 (77\%), which was subjected to dihydroxylation in the presence of the Corey dihydroquinidine ligand 29 , thus producing the $(R)$-diol 30 (58\%, $78 \%$ brsm, $88 \%$ ee as determined by Mosher ester analysis). ${ }^{15}$ Diol 30 was subsequently converted into the $(S)$ epoxide 31 (93\%) via mesylation and potassium carbonate mediated cyclization. The allyl protecting group was selectively removed by reaction with dimethylbarbituric acid 32 catalyzed by $\mathrm{Pd}\left(\mathrm{PPh}_{3}\right)_{4}$ to provide epoxide $33(91 \%) .{ }^{16}$ After screening a variety of different Lewis acids with epoxide 33, it was found that ferric chloride hydrate was a superior Lewis acid catalyst to boron trifluoride etherate, ${ }^{9 e}$ which we previously reported for this cyclization of epoxide 33 to provide meroterpenoid 34 . The use of boron trifluoride etherate often led to the formation of undesired byproducts such as bicyclic ethers and partially cyclized products. Such side reactions and irreproducibility were substantially suppressed using ferric chloride hydrate. ${ }^{17}$ Thus, treatment of epoxide 33 with $\mathrm{FeCl}_{3} \cdot 6 \mathrm{H}_{2} \mathrm{O}$ resulted in biomimetic cationic cyclization to give meroterpeonoid 34 (56\%, $92 \%$ ee as determined by chiral HPLC) as a single diastereoisomer. Saponification of meroterpenoid 34 gave acid $35(69 \%),{ }^{13}$ which was subjected to acetylation to provide diacetate 36. Subsequent selective deacetylation of the phenolic acetate gave (+)-hongoquercin B (66\%) with an overall yield of $3.7 \%$ over 11 steps. The analytical data for this synthetic material are in full agreement with that reported for the isolated natural product. ${ }^{1}$

Additional meroterpenoids analogues were prepared via epoxidation (Scheme 7) and halogenations (Scheme 8). First, the geranyl-substituted resorcylate $\mathbf{2 4}$ was protected as its silyl ether $37(76 \%)$ and epoxidized with the dioxirane derived from the Shi chiral ketone 38 to give epoxide 39 (69\%). ${ }^{18}$ Subsequent deprotection gave epoxide 40 (92\%), which was cyclized using boron trifluoride etherate to give meroterpenoid 41 (77\%, 84\% ee as determined by chiral HPLC) as a single diastereoisomer. The $(S)$-enantiomer of meroterpenoid $\mathbf{4 1}$ (98\% ee as determined by chiral HPLC) was obtained by recrystallization to enhance chiral purity. Second, we examined the halonium-induced polyene cyclization of the resorcylates to produce additional analogues (Scheme 8). ${ }^{19}$ Reaction of the geranyl resorcylate $\mathbf{2 4}$ with the Snyder reagents $\mathrm{Et}_{2} \mathrm{SBr}$. $\mathrm{SbCl}_{5} \mathrm{Br}(\mathrm{BDSB}, 46)$ and $\left(\mathrm{Et}_{2} \mathrm{SI}\right)_{2} \mathrm{Cl} \cdot \mathrm{SbCl}_{6}$ (IDSI, 47) resulted in bromo- and iodo-cyclizations to produce the racemic bromo-meroterpenoid $\mathbf{4 2}$ (64\%) and racemic iodo-meroterpenoid $43(88 \%)$ as single diastereoisomers, respectively. Racemic bromide 44 (45\%, 2:1 dr) and racemic iodide 45 $(54 \%, 2: 1 \mathrm{dr})$ were also successfully synthesized from farnesylsubstituted resorcylate 14 using the BDSB- (46) and IDSImediated (47) halocyclizations. ${ }^{20}$

The 2,2-dimethyl-1,3-benzodioxan-4-one moiety of the meroterpenoids intermediates was also used in alternative derivatization reactions (Scheme 9). Thus, reaction of the geranyl-substituted resorcylate $\mathbf{2 4}$ with boron trifluoride etherate at $25{ }^{\circ} \mathrm{C}$ gave the racemic meroterpenoid 48 (89\%, $3: 1 \mathrm{dr}$ ), and the desired pure trans-fused ring product was obtained by recrystallization from $n$-hexane. ${ }^{21}$ Saponification ${ }^{13}$ of meroterpenoid 48 gave racemic carboxylic acid 49 (69\%), while hydrolytic decarboxylation gave racemic phenol $\mathbf{5 0}$ (97\%). Furthermore, reduction of meroterpenoid 48 with $\mathrm{LiAlH}_{4}$ gave racemic diol 51 (95\%), and racemic Weinreb amide 52 (96\%) was obtained following Grignard reagent mediated amidation. ${ }^{22}$ 
Scheme 6. Total Synthesis of (+)-Hongoquercin B (2)
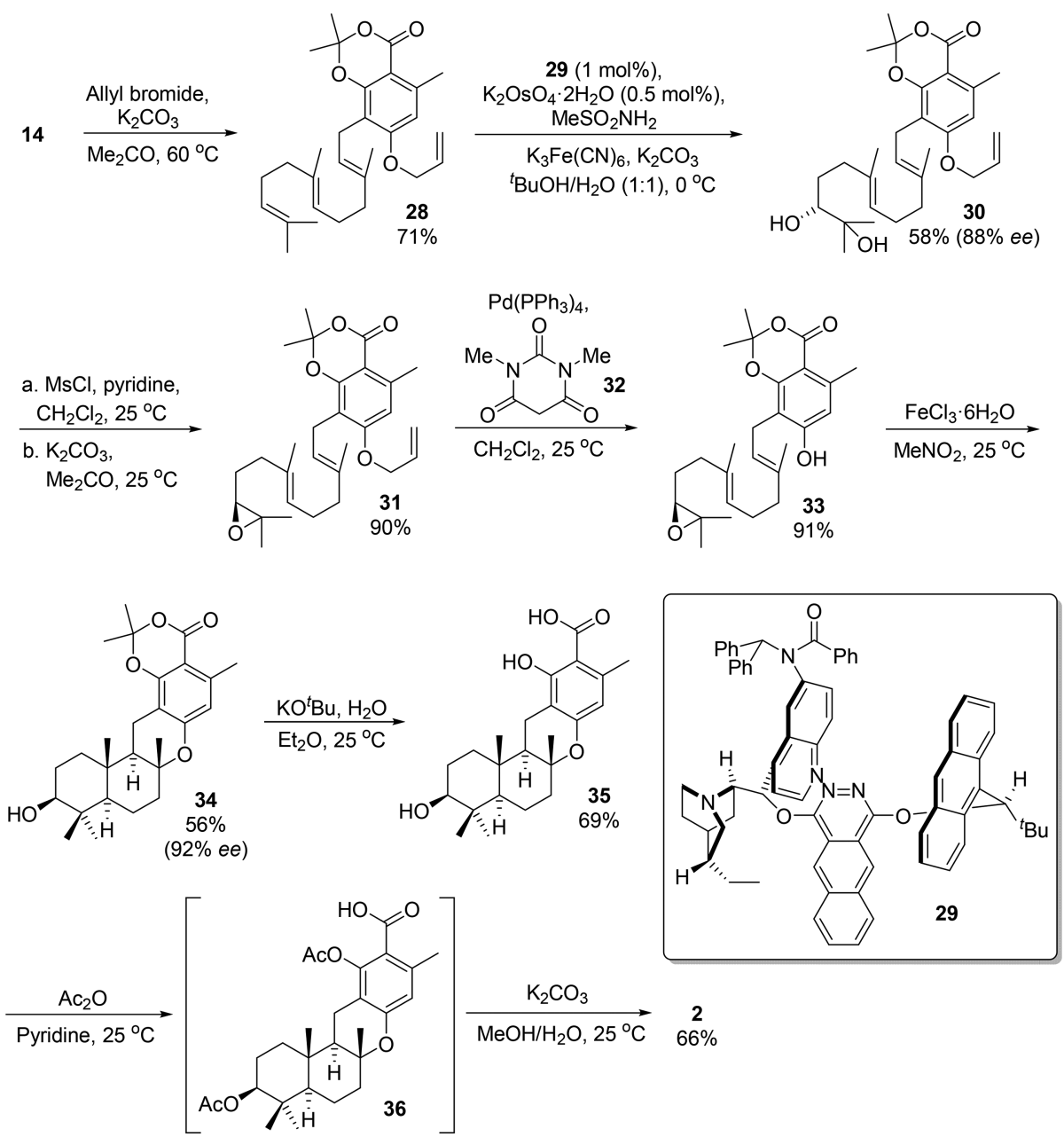

Scheme 7. Synthesis of Meroterpenoid 41

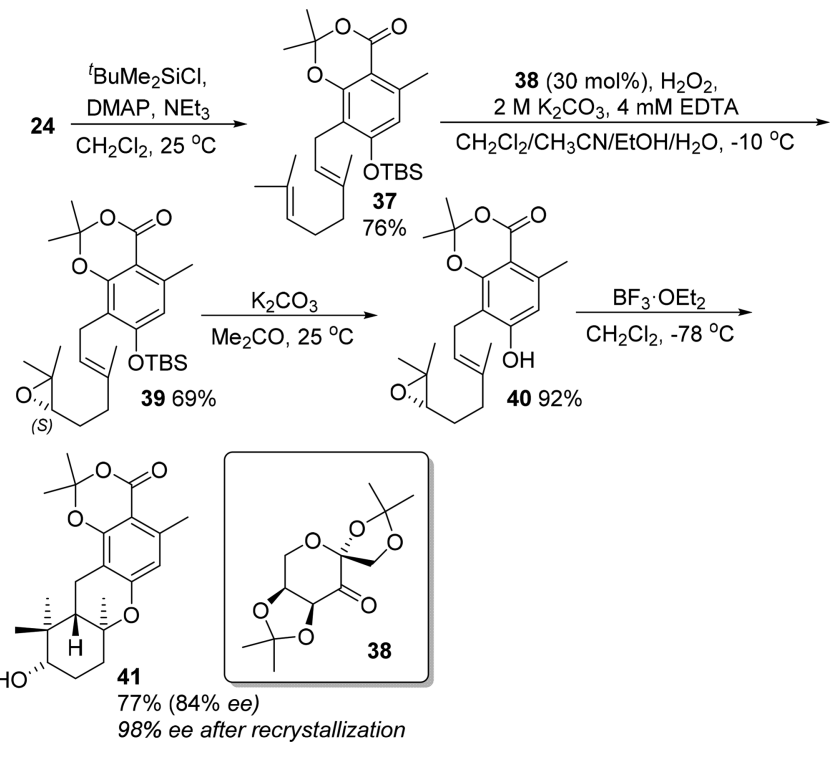

\section{CONCLUSION}

In conclusion, the total syntheses of (+)-hongoquercins A (1) and B (2) were completed in five and eleven steps, respectively with an overall yield of $20 \%$ and $3.7 \%$ via a dual biomimetic
Scheme 8. Halocyclizations To Produce Meroterpenoids $42-45^{20}$

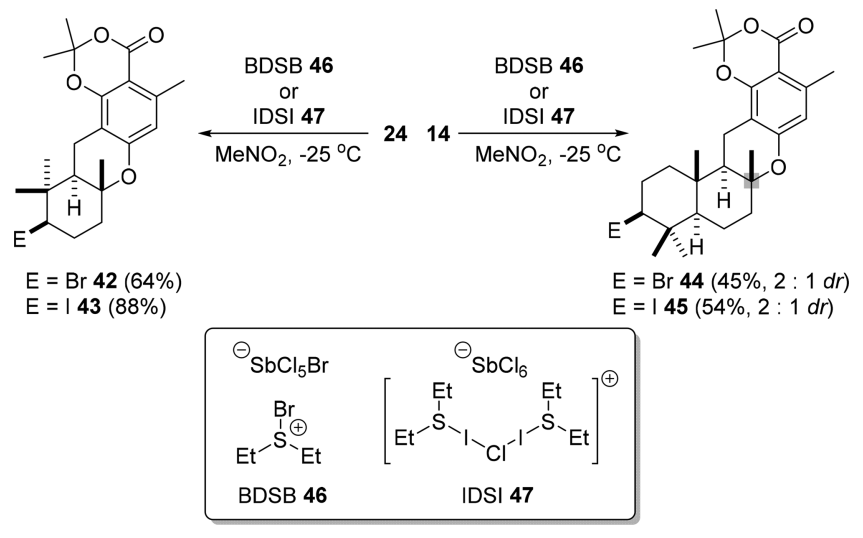

approach involving sequential polyketide and late-stage electrophile-mediated polyene cyclizations. Several analogues were synthesized by epoxidation or bromo- and iodocyclizations. The meroterpenoids were additionally functionalized using saponification, hydrolytic decarboxylation, reduction, and Grignard reagent mediated amidation reactions. Further studies on the synthesis of novel meroterpenoids adopting such dual biomimetic approach are ongoing in our laboratory. 
Scheme 9. Synthesis and Functionalization of Meroterpenoid 48

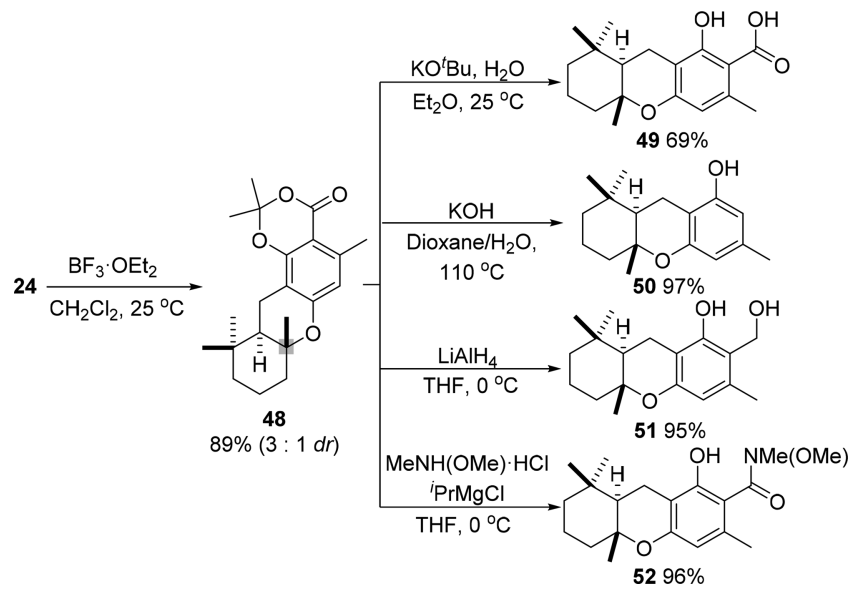

\section{EXPERIMENTAL SECTION}

General Methods. All reagents and solvents were used directly without further purification unless otherwise stated. The preparation of malonate and dioxinone acid was performed according to the method of Barrett et al. ${ }^{10 \mathrm{~b}}$ Binol 25 and binol 26 were prepared, respectively, according to the procedures reported by Corey et al. ${ }^{11}$ and Yamamoto et al. ${ }^{12}$ Dihydroquinidine ligand 29 was prepared according to the procedure reported by Corey et al. ${ }^{15} \mathrm{Et}_{2} \mathrm{SBr} \cdot \mathrm{SbCl}_{5} \mathrm{Br}$ $(\mathrm{BDSB}, 46)$ and $\left(\mathrm{Et}_{2} \mathrm{SI}\right)_{2} \mathrm{Cl} \cdot \mathrm{SbCl}_{6}$ (IDSI, 47) were prepared according to the method published by Snyder et al. ${ }^{19}$ All solvents were purified and dried by distillation under an atmosphere of $\mathrm{N}_{2}$ before use. The chiral ketone $\mathbf{3 8}$ was prepared from L-fructose according to the established method by Shi et al. ${ }^{18} \mathrm{Et}_{2} \mathrm{O}$ and THF were redistilled from $\mathrm{Na}-\mathrm{Ph}_{2} \mathrm{CO} . \mathrm{CH}_{2} \mathrm{Cl}_{2}, \mathrm{Et}_{3} \mathrm{~N}, \mathrm{MeOH},{ }^{i} \mathrm{PrNO}_{2}$, $\mathrm{MeNO}_{2}$, and pyridine were redistilled from $\mathrm{CaH}_{2}$, and $\mathrm{PhMe}$ was redistilled from $\mathrm{Na}$. All air- and moisture-sensitive reactions were carried out under an atmosphere of $\mathrm{N}_{2}$ using standard Schlenk techniques in oven-dried glassware equipped with a magnetic stirring bar. The progress of reactions was monitored by analytical thin-layer chromatography (TLC) on silica gel coated aluminum oxide $\mathrm{F}_{254}$ plates. Developed TLC was visualized under UV light and stained with acidic vanillin solution. Flash column chromatography was performed by employing silica gel $60 \AA$, particle size $40-63 \mu \mathrm{m}$. The enantiomeric excesses of the compounds were determined by chiral HPLC analysis on Chiralpak IE column with $n$-hexane and ${ }^{i} \mathrm{PrOH}$ as the mobile phase. All ${ }^{1} \mathrm{H}$ and proton-decoupled ${ }^{13} \mathrm{C}$ NMR spectra were recorded at 400 and $101 \mathrm{MHz}$, respectively, at ambient temperature in deuterated solvent as noted. NMR spectra were referenced to residual solvent peaks $\left(\mathrm{CDCl}_{3}: \delta=7.26\right.$ for ${ }^{1} \mathrm{H}$ NMR and $\delta=77.0$ for ${ }^{13} \mathrm{C}$ NMR; $\mathrm{CD}_{3} \mathrm{OD} \delta=3.31$ and 4.87 for ${ }^{1} \mathrm{H}$ NMR and $\delta=49.0$ for ${ }^{13} \mathrm{C}$ NMR; $\left(\mathrm{CD}_{3}\right)_{2} \mathrm{CO}: \delta=2.05$ for ${ }^{1} \mathrm{H} \mathrm{NMR}$ and $\delta=$ 29.8 for ${ }^{13} \mathrm{C}$ NMR) and chemical shifts were reported in ppm. IR spectra are reported in $\mathrm{cm}^{-1}$. Optical rotations were recorded with a polarimeter with the specified concentration and temperature. Mass spectra were obtained from the Imperial College Mass Spectrometry Service with the use of TOF and magnetic analyzers for ESI and EI techniques, respectively. Melting points are uncorrected. X-ray diffraction data were recorded at the Imperial College X-ray Crystallography Facility.

General Procedures for the Synthesis of Dioxinone $\boldsymbol{\beta}$-Keto Esters. 2-Phenyl-1,3-dioxane-4,6-dione $(6.91 \mathrm{~g}, 36.0 \mathrm{mmol})$, DCC (7.43 g, $36.0 \mathrm{mmol})$, and DMAP (4.40 g, $36.0 \mathrm{mmol})$ were dissolved in $\mathrm{CH}_{2} \mathrm{Cl}_{2}(300 \mathrm{~mL})$, and the resulting mixture was stirred for $5 \mathrm{~min}$ at $25{ }^{\circ} \mathrm{C}$. 2-(2,2-Dimethyl-4-oxo-4H-1,3-dioxin-6-yl)acetic acid (6.70 g, $36.0 \mathrm{mmol}$ ) was added with stirring at $25^{\circ} \mathrm{C}$. After $18 \mathrm{~h}$, the mixture was cooled to $0{ }^{\circ} \mathrm{C}$, and the insoluble solid was filtered off and washed with $\mathrm{CH}_{2} \mathrm{Cl}_{2}(15 \mathrm{~mL})$. The filtrate was washed with aqueous $\mathrm{HCl}(1 \mathrm{M} ; 2 \times 200 \mathrm{~mL})$, the two phases were separated, and the organic layer was dried $\mathrm{MgSO}_{4}$ ), filtered, and concentrated under reduced pressure to give the crude delicate dioxane-4,6-dione keto dioxinone 19 as a yellow foam, which was used directly without further purification. Crude dioxane-4,6-dione keto dioxinone 19 and geraniol (18) or trans,trans-farnesol (17) $(20.0 \mathrm{mmol})$ were dissolved in $\mathrm{PhMe}(150 \mathrm{~mL})$ and stirred at $55{ }^{\circ} \mathrm{C}$ for $4 \mathrm{~h}$. The reaction mixture was concentrated under reduced pressure, and the brown residue was chromatographed (pentane/EtOAc 9:1-4:1) to provide the dioxinone $\beta$-keto esters 20 and $\mathbf{2 1}$, respectively.

(E)-3,7-Dimethylocta-2,6-dienyl 4-(2,2-dimethyl-4-oxo-4H-1,3dioxin-6-yl)-3-oxobutanoate (20). Dioxinone $\beta$-keto ester 20 (6.92 g, $19.0 \mathrm{mmol}, 95 \%)$, prepared from geraniol $(18)(3.47 \mathrm{~mL}, 20.0$ mmol), was obtained as a pale yellow oil: $R_{f} 0.20$ (pentane/EtOAc 4:1); ${ }^{1} \mathrm{H}$ NMR $\left(400 \mathrm{MHz}, \mathrm{CDCl}_{3}\right) \delta 5.36(\mathrm{~s}, 1 \mathrm{H}), 5.34-5.30(\mathrm{~m}$, $1 \mathrm{H}), 5.09-5.04(\mathrm{~m}, 1 \mathrm{H}), 4.67(\mathrm{~d}, J=7.2 \mathrm{~Hz}, 2 \mathrm{H}), 3.51(\mathrm{~s}, 2 \mathrm{H}), 3.50$ (s, 2H), 2.14-2.01 (m, 4H), $1.71(\mathrm{~s}, 6 \mathrm{H}), 1.68(\mathrm{~s}, 3 \mathrm{H}), 1.67(\mathrm{~s}, 3 \mathrm{H})$, $1.59(\mathrm{~s}, 3 \mathrm{H}) ;{ }^{13} \mathrm{C}\left\{{ }^{1} \mathrm{H}\right\}$ NMR $\left(101 \mathrm{MHz}, \mathrm{CDCl}_{3}\right) \delta 195.6,166.3$, $163.5,160.4,143.6,132.0,123.5,117.3,107.3,97.1,62.6,49.1,46.9$, 39.5, 26.2, 25.6, 25.0, 17.7, 16.5; IR $\nu_{\max }$ (neat) 1720, 1375, 1272, $1201,1015 \mathrm{~cm}^{-1}$; HRMS (ESI) $\mathrm{m} / z[\mathrm{M}+\mathrm{H}]^{+}$calcd for $\mathrm{C}_{20} \mathrm{H}_{29} \mathrm{O}_{6}$ 365.1964, found 365.1977 .

(2E,6E)-3,7,11-Trimethyldodeca-2,6,10-trienyl 4-(2,2-dimethyl-4oxo-4H-1,3-dioxin-6-yl)-3-oxobutanoate (21). Dioxinone $\beta$-keto ester $21(6.81 \mathrm{~g}, 15.7 \mathrm{mmol}, 79 \%)$, prepared from trans,trans-farnesol (17) $(5.02 \mathrm{~mL}, 20.0 \mathrm{mmol})$, was obtained as a pale yellow oil: $R_{f} 0.36$ (pentane/ $\left.\mathrm{Et}_{2} \mathrm{O} 1: 1\right) ;{ }^{1} \mathrm{H}$ NMR $\left(400 \mathrm{MHz}, \mathrm{CDCl}_{3}\right) \delta 5.34(\mathrm{~s}, 1 \mathrm{H})$, $5.33-5.28(\mathrm{~m}, 1 \mathrm{H}), 5.11-5.02(\mathrm{~m}, 2 \mathrm{H}), 4.65(\mathrm{~d}, J=7.2 \mathrm{~Hz}, 2 \mathrm{H})$, $3.49(\mathrm{~s}, 2 \mathrm{H}), 3.48(\mathrm{~s}, 2 \mathrm{H}), 2.13-1.99(\mathrm{~m}, 6 \mathrm{H}), 1.99-1.91(\mathrm{~m}, 2 \mathrm{H})$, $1.69(\mathrm{~s}, 6 \mathrm{H}), 1.66(\mathrm{~s}, 3 \mathrm{H}), 1.65(\mathrm{~s}, 3 \mathrm{H}), 1.57(\mathrm{~s}, 6 \mathrm{H}) ;{ }^{13} \mathrm{C}\left\{{ }^{1} \mathrm{H}\right\} \mathrm{NMR}$ $\left(101 \mathrm{MHz}, \mathrm{CDCl}_{3}\right) \delta 195.6,166.3,163.5,160.4,143.5,135.5,131.2$, $124.2,123.4,117.3,107.2,97.0,62.5,49.0,46.9,39.6,39.4,26.6,26.1$, 25.6, 24.9, 17.6, 16.4, 15.9; IR $\nu_{\max }$ (neat) 2922, 1722, 1639, 1375, $1272,1202,1015,901,806 \mathrm{~cm}^{-1}$; HRMS (ESI) $\mathrm{m} / z[\mathrm{M}+\mathrm{H}]^{+}$calcd for $\mathrm{C}_{25} \mathrm{H}_{37} \mathrm{O}_{6} 433.2590$, found 433.2598. Anal. Calcd for $\mathrm{C}_{25} \mathrm{H}_{36} \mathrm{O}_{6}$ : C, $69.42 ; \mathrm{H}, 8.39$. Found: C, 69.49; H, 8.24.

General Procedure for the Synthesis of Resorcylates 14 and 24. $\mathrm{MgCl}_{2}(476 \mathrm{mg}, 5.00 \mathrm{mmol})$ and pyridine $(0.810 \mathrm{~mL}, 10.0$ $\mathrm{mmol})$ were added with stirring to $\beta$-keto ester 20 or $21(5.00 \mathrm{mmol})$ in $\mathrm{CH}_{2} \mathrm{Cl}_{2}(25 \mathrm{~mL})$ at $0{ }^{\circ} \mathrm{C}$. After $15 \mathrm{~min}, \mathrm{AcCl}(0.540 \mathrm{~mL}, 7.50$ $\mathrm{mmol}$ ) was added dropwise, and the reaction mixture was further stirred for $1 \mathrm{~h}$ at $0{ }^{\circ} \mathrm{C}$. The reaction was quenched by addition of saturated aqueous $\mathrm{NH}_{4} \mathrm{Cl}(20 \mathrm{~mL})$, and the $\mathrm{pH}$ was adjusted to $\sim 2$ with aqueous $\mathrm{HCl}(1 \mathrm{M})$. The two phases were separated, and the aqueous layer was extracted with EtOAc $(3 \times 50 \mathrm{~mL})$. The combined organic layers were dried $\left(\mathrm{MgSO}_{4}\right)$, filtered, and concentrated under reduced pressure to give the crude dioxinone $\beta, \delta$-diketo ester $\mathbf{2 2}$ or 23. $\mathrm{P}(2 \text {-furyl })_{3}(232 \mathrm{mg}, 1.00 \mathrm{mmol})$ and $\mathrm{Pd}_{2} \mathrm{dba}_{3}(229 \mathrm{mg}, 0.250$ $\mathrm{mmol}$ ) were added sequentially with stirring to this crude material in THF $(30 \mathrm{~mL})$ at $25{ }^{\circ} \mathrm{C}$. After $1 \mathrm{~h}, \mathrm{CsOAc}(2.88 \mathrm{~g}, 15.0 \mathrm{mmol})$ in ${ }^{i} \mathrm{PrOH}(30 \mathrm{~mL})$ was added, and the resulting mixture was stirred for an dditional $1.5 \mathrm{~h}$. The reaction was quenched with aqueous $\mathrm{HCl}(1$ $\mathrm{M} ; 30 \mathrm{~mL}$ ), the two phases were separated, and the aqueous layer was extracted with $\mathrm{CH}_{2} \mathrm{Cl}_{2}(3 \times 5 \mathrm{~mL})$. The combined organic layers were dried $\left(\mathrm{MgSO}_{4}\right)$, filtered, concentrated under reduced pressure, and chromatographed (pentane/EtOAc 19:1-10:1) to give resorcylate 24 or 14.

(E)-8-(3,7-Dimethylocta-2,6-dienyl)-7-hydroxy-2,2,5-trimethyl4H-benzo[d][1,3]dioxin-4-one (24). Resorcylate 24 (1.03 g, 3.02 $\mathrm{mmol}, 60 \%$ over two steps), prepared from dioxinone $\beta$-keto ester 20 $(1.83 \mathrm{~g}, 5.00 \mathrm{mmol})$, was obtained as a white solid. An analytically pure sample was obtained by recrystallization from $\mathrm{MeNO}_{2}: R_{f} 0.37$ (pentane/EtOAc 4:1); mp 102.9-103.0 ${ }^{\circ} \mathrm{C} ;{ }^{1} \mathrm{H}$ NMR (400 MHz, $\left.\mathrm{CDCl}_{3}\right) \delta 6.42(\mathrm{~s}, 1 \mathrm{H}), 6.06(\mathrm{~s}, 1 \mathrm{H}), 5.19(\mathrm{t}, J=7.4 \mathrm{~Hz}, 1 \mathrm{H}), 5.04(\mathrm{t}$, $J=6.3 \mathrm{~Hz}, 1 \mathrm{H}), 3.33(\mathrm{~d}, J=7.2 \mathrm{~Hz}, 2 \mathrm{H}), 2.59(\mathrm{~s}, 3 \mathrm{H}), 2.14-1.98(\mathrm{~m}$, $4 \mathrm{H}), 1.79(\mathrm{~s}, 3 \mathrm{H}), 1.69(\mathrm{~s}, 6 \mathrm{H}), 1.67(\mathrm{~s}, 3 \mathrm{H}), 1.59(\mathrm{~s}, 3 \mathrm{H}) ;{ }^{13} \mathrm{C}\left\{{ }^{1} \mathrm{H}\right\}$ NMR $\left(126 \mathrm{MHz}, \mathrm{CDCl}_{3}\right) \delta 161.2,160.2,156.0,142.8,138.5,132.0$, $123.7,120.8,113.7,112.8,105.3,104.8,39.7,26.4,25.7,25.6,22.0$, 21.9, 17.7, 16.2; IR $\nu_{\max }$ (neat) 3199, 1694, 1608, 1300, 1282, 1210, $1177,1106,1045,754 \mathrm{~cm}^{-1}$; HRMS (ESI) $\mathrm{m} / z[\mathrm{M}+\mathrm{H}]^{+}$calcd for $\mathrm{C}_{21} \mathrm{H}_{29} \mathrm{O}_{4}$ 345.2066, found 345.2067. Anal. Calcd for $\mathrm{C}_{21} \mathrm{H}_{28} \mathrm{O}_{4}$ : C, 73.32; H, 8.19. Found: C, 73.35; H, 8.31. 
7-Hydroxy-2,2,5-trimethyl-8-((2E,6E)-3,7,11-trimethyldodeca2,6,10-trienyl)-4H-benzo[d][1,3]dioxin-4-one (14). Resorcylate 14 ( $1.14 \mathrm{~g}, 2.75 \mathrm{mmol}, 55 \%$ over two steps), prepared from dioxinone $\beta$ keto ester 21 ( $2.17 \mathrm{~g}, 5.00 \mathrm{mmol})$, was obtained as a yellow oil, which solidified upon standing: $R_{f} 0.24$ (pentane/EtOAc 9:1); mp $72.2{ }^{\circ} \mathrm{C}$; ${ }^{1} \mathrm{H}$ NMR $\left(400 \mathrm{MHz}, \mathrm{CDCl}_{3}\right) \delta 6.41(\mathrm{~s}, 1 \mathrm{H}), 6.15(\mathrm{~s}, 1 \mathrm{H}), 5.19(\mathrm{t}, J=$ $6.7 \mathrm{~Hz}, 1 \mathrm{H}), 5.13-5.00(\mathrm{~m}, 2 \mathrm{H}), 3.32(\mathrm{~d}, J=7.2 \mathrm{~Hz}), 2.58(\mathrm{~s}, 3 \mathrm{H})$, $2.14-1.90(\mathrm{~m}, 8 \mathrm{H}), 1.79(\mathrm{~s}, 3 \mathrm{H}), 1.68(\mathrm{~s}, 6 \mathrm{H}), 1.67(\mathrm{~s}, 3 \mathrm{H}), 1.58(\mathrm{~s}$, $6 \mathrm{H}) ;{ }^{13} \mathrm{C}\left\{{ }^{1} \mathrm{H}\right\} \mathrm{NMR}\left(101 \mathrm{MHz}, \mathrm{CDCl}_{3}\right) \delta 161.0,160.0,156.0,142.8$, $138.6,135.6,131.3,124.3,123.5,120.8,113.6,112.7,105.3,104.8$, $39.7,39.7,26.7,26.3,25.7,25.7,22.0,21.9,17.7,16.2,16.0$; IR $\nu_{\max }$ (neat) $3209,2971,2926,1738,1704,1964,1606,1590,1376,1284$, $1217,1170,1107,1046,858,751 \mathrm{~cm}^{-1}$; HRMS (ESI) $m / z[\mathrm{M}+\mathrm{H}]^{+}$ calcd for $\mathrm{C}_{26} \mathrm{H}_{37} \mathrm{O}_{4}$ 413.2692, found 413.2680. Anal. Calcd for $\mathrm{C}_{26} \mathrm{H}_{36} \mathrm{O}_{4}$ : C, 75.69; $\mathrm{H}, 8.80$. Found: $\mathrm{C}, 75.56 ; \mathrm{H}, 8.96$.

(7aR, 9aS, 13aS, 13bR)-2,2,5,7a, 10,10,13a-Heptamethyl-7a,8,9a,$10,11,12,13,13 a, 13 b, 14$-decahydro-4H,9H-benzo[a][1,3]dioxino[5,4-j]xanthen-4-one (27). $\mathrm{SnCl}_{4}$ in heptane $(1 \mathrm{M}$; $0.750 \mathrm{~mL}, 0.750$ $\mathrm{mmol}$ ) was added with stirring to binol 26 (463 $\mathrm{mg}, 0.900 \mathrm{mmol})$ in $\mathrm{PhMe}(9 \mathrm{~mL})$ at $25^{\circ} \mathrm{C}$. After $10 \mathrm{~min}$, the mixture was cooled to -78 ${ }^{\circ} \mathrm{C}$, when resorcylate $14(124 \mathrm{mg}, 0.300 \mathrm{mmol})$ in $\mathrm{PhMe}(1.5 \mathrm{~mL})$ was added dropwise and the reaction mixture was further stirred for $48 \mathrm{~h}$ at $-78{ }^{\circ} \mathrm{C}$. The reaction was quenched with $\mathrm{NaHCO}_{3}(15 \mathrm{~mL})$ and the mixture diluted with $\mathrm{Et}_{2} \mathrm{O}(10 \mathrm{~mL})$. The two phases were separated, and the aqueous layer was extracted with $\mathrm{CH}_{2} \mathrm{Cl}_{2}(4 \times 15$ $\mathrm{mL})$. The combined organic layers were dried $\left(\mathrm{MgSO}_{4}\right)$, filtered, concentrated, and chromatographed (pentane/ $\mathrm{Et}_{2} \mathrm{O} 9: 1$ to $\mathrm{PhMe}$ / $\mathrm{Et}_{2} \mathrm{O}$ 12:1) to recover the binol 26. The crude mixture of products was then dissolved in 2-nitropropane $(6 \mathrm{~mL})$ and cooled to $-78{ }^{\circ} \mathrm{C}$. $\mathrm{SnCl}_{4}$ in heptane $(1 \mathrm{M} ; 0.750 \mathrm{~mL}, 0.750 \mathrm{mmol})$ and $\mathrm{CF}_{3} \mathrm{COOH}$ $(0.230 \mathrm{~mL}, 3.00 \mathrm{mmol})$ were sequentially added dropwise with stirring at $-78{ }^{\circ} \mathrm{C}$. After $24 \mathrm{~h}$, the reaction was quenched with saturated aqueous $\mathrm{NaHCO}_{3}(10 \mathrm{~mL})$ and diluted with $\mathrm{Et}_{2} \mathrm{O}(10 \mathrm{~mL})$. The two phases were separated, and the aqueous layer was extracted with $\mathrm{Et}_{2} \mathrm{O}(4 \times 15 \mathrm{~mL})$. The combined organic layers were dried $\left(\mathrm{MgSO}_{4}\right)$, filtered, concentrated, and chromatographed (pentane/ $\left.\mathrm{Et}_{2} \mathrm{O} 9: 1\right)$ to provide meroterpenoid $27(78 \mathrm{mg}, 0.189 \mathrm{mmol}, 63 \%$, $80 \% \mathrm{dr}, 90 \%$ ee, measured by chiral HPLC, Chiralpak IE column, $n$ hexane $/{ }^{i} \mathrm{PrOH}$ 19:1, $5 \mathrm{~mL} / \mathrm{min}, t_{\mathrm{R}}=15.9[(+)$-enantiomer], 14.9 $[(-)$-enantiomer $] \mathrm{min})$ as a colorless oil containing a mixture of diastereoisomers. An analytical sample was purified by preparative chiral HPLC: $R_{f} 0.75$ (pentane/EtOAc 9:1); $[\alpha]_{\mathrm{D}}^{20}+80.3(c 0.57$, $\mathrm{MeOH}) ;{ }^{1} \mathrm{H}$ NMR $\left(500 \mathrm{MHz}, \mathrm{CDCl}_{3}\right) \delta 6.33(\mathrm{~s}, 1 \mathrm{H}), 2.57(\mathrm{~s}, 3 \mathrm{H})$, $2.53(\mathrm{dd}, 1 \mathrm{H}), 2.24(\mathrm{dd}, J=16.8,13.2 \mathrm{~Hz}, 1 \mathrm{H}), 2.08(\mathrm{dt}, J=12.5,3.2$ $\mathrm{Hz}, 1 \mathrm{H}), 1.82-1.77(\mathrm{~m}, 1 \mathrm{H}), 1.77-1.74(\mathrm{~m}, 1 \mathrm{H}), 1.72(\mathrm{~s}, 3 \mathrm{H}), 1.69$ $(\mathrm{s}, 3 \mathrm{H}), 1.68-1.65(\mathrm{~m}, 1 \mathrm{H}), 1.65-1.62(\mathrm{~m}, 1 \mathrm{H}), 1.53(\mathrm{~d}, J=5.3 \mathrm{~Hz}$, $1 \mathrm{H}), 1.52-1.45(\mathrm{~m}, 1 \mathrm{H}), 1.45-1.39(\mathrm{~m}, 1 \mathrm{H}), 1.39-1.34(\mathrm{~m}, 1 \mathrm{H})$, $1.19(\mathrm{~s}, 3 \mathrm{H}), 1.16(\mathrm{dd}, J=13.5,4.3 \mathrm{~Hz}, 1 \mathrm{H}), 1.02(\mathrm{dd}, J=12.2,2.3$ $\mathrm{Hz}, 1 \mathrm{H}), 0.98(\mathrm{dd}, J=12.8,3.8 \mathrm{~Hz}, 1 \mathrm{H}), 0.91(\mathrm{~s}, 6 \mathrm{H}), 0.85(\mathrm{~s}, 3 \mathrm{H})$; ${ }^{13} \mathrm{C}\left\{{ }^{1} \mathrm{H}\right\}$ NMR $\left(101 \mathrm{MHz}, \mathrm{CDCl}_{3}\right) \delta 160.9,158.7,156.1,142.0$, 114.3, 108.3, 104.8, 103.9, 78.4, 56.1, 51.3, 41.8, 40.8, 39.2, 36.9, 33.4, 33.2, 26.2, 25.5, 22.0, 21.6, 20.7, 19.7, 18.5, 16.5, 14.9; IR $\nu_{\max }$ (neat) 2928, 2867, 1728, 1616, 1575, 1452, 1388, 1285, $1127 \mathrm{~cm}^{-1}$; HRMS (ESI) $m / z[\mathrm{M}+\mathrm{H}]^{+}$calcd for $\mathrm{C}_{26} \mathrm{H}_{37} \mathrm{O}_{4} 413.2696$, found 413.2698.

(+)-Hongoquercin $A$ [(4aS,6aR,12aR,12bS)-11-Hydroxy$4,4,6 a, 9,12 b$-pentamethyl-1,3,4,4a,5,6,6a, 12,12a,12b-decahydro$2 \mathrm{H}$-benzo[a]xanthene-10-carboxylic Acid (1)]. $\mathrm{H}_{2} \mathrm{O}(7 \mu \mathrm{L}, 0.383$ $\mathrm{mmol})$ was added with stirring to a suspension of meroterpenoid 27 (79.0 mg, $0.191 \mathrm{mmol})$ and $\mathrm{KO}^{t} \mathrm{Bu}(172 \mathrm{mg}, 1.53 \mathrm{mmol})$ in $\mathrm{Et}_{2} \mathrm{O}(3$ $\mathrm{mL}$ ) at $25{ }^{\circ} \mathrm{C}$. After $2 \mathrm{~h}$, ice was added until two layers were formed, and the two phases were separated. The organic layer was extracted with $\mathrm{H}_{2} \mathrm{O}(5 \times 5 \mathrm{~mL})$, and the combined aqueous layers were acidified with $\mathrm{HCl}(4 \mathrm{M})$ to $\mathrm{pH} \sim 1$. The two phases were separated, and the aqueous layer was extracted with $\mathrm{Et}_{2} \mathrm{O}(5 \times 5 \mathrm{~mL})$. The combined organic layers were dried $\left(\mathrm{MgSO}_{4}\right)$, filtered, concentrated, and chromatographed (pentane/EtOAc/AcOH 9:1:0.01) to afford (+)-hongoquercin A (1) (53 mg, $0.142 \mathrm{mmol}, 75 \%)$ as white solid. An analytical sample was prepared by recrystallization (pentane/ $\mathrm{CH}_{2} \mathrm{Cl}_{2}$ ): $R_{f} 0.25$ (pentane/EtOAc/AcOH 9:1:0.01); mp 146.1$148.5^{\circ} \mathrm{C} ;[\alpha]_{\mathrm{D}}^{24}+90.5$ (c 0.57, MeOH); ${ }^{1} \mathrm{H} \mathrm{NMR}\left(400 \mathrm{MHz}, \mathrm{CDCl}_{3}\right)$ $\delta 11.86(\mathrm{~s}, 1 \mathrm{H}), 6.21(\mathrm{~s}, 1 \mathrm{H}), 2.69(\mathrm{dd}, J=16.9,5.0 \mathrm{~Hz}, 1 \mathrm{H}), 2.51(\mathrm{~s}$, $3 \mathrm{H}), 2.29(\mathrm{dd}, J=16.9,13.2 \mathrm{~Hz}, 1 \mathrm{H}), 2.08(\mathrm{dt}, J=12.5,3.1 \mathrm{~Hz}, 1 \mathrm{H})$, $1.86-1.72(\mathrm{~m}, 2 \mathrm{H}), 1.69(\mathrm{dd}, J=13.1,4.8 \mathrm{~Hz}, 1 \mathrm{H}), 1.66-1.58(\mathrm{~m}$, $1 \mathrm{H}), 1.55(\mathrm{dd}, J=13.1,5.0 \mathrm{~Hz}, 1 \mathrm{H}), 1.52-1.45(\mathrm{~m}, 1 \mathrm{H}), 1.44-1.42$ (m, $1 \mathrm{H}), 1.42-1.34(\mathrm{~m}, 1 \mathrm{H}), 1.20(\mathrm{~s}, 3 \mathrm{H}), 1.15(\mathrm{dd}, J=13.4,4.0 \mathrm{~Hz}$, $1 \mathrm{H}), 1.03(\mathrm{dd}, J=12.2,2.2 \mathrm{~Hz}, 1 \mathrm{H}), 1.00-0.94(\mathrm{~m}, 1 \mathrm{H}), 0.92(\mathrm{~s}$, $3 \mathrm{H}), 0.91$ (s, 3H), 0.85 (s, 3H). ${ }^{1} \mathrm{H}$ NMR $\left(500 \mathrm{MHz}, \mathrm{CD}_{3} \mathrm{OD}\right) \delta$ $6.11(\mathrm{~s}, 1 \mathrm{H}), 2.65(\mathrm{dd}, J=16.9,5.0 \mathrm{~Hz}, 1 \mathrm{H}), 2.46(\mathrm{~s}, 3 \mathrm{H}), 2.27(\mathrm{dd}, J$ $=16.7,13.2 \mathrm{~Hz}, 1 \mathrm{H}), 2.05(\mathrm{dt}, J=12.5,3.2 \mathrm{~Hz}, 1 \mathrm{H}), 1.81-1.76(\mathrm{~m}$, $2 \mathrm{H}), 1.72(\mathrm{dt}, J=13.7,3.6 \mathrm{~Hz}, 1 \mathrm{H}), 1.69-1.61(\mathrm{~m}, 1 \mathrm{H}), 1.52(\mathrm{dd}, J=$ $13.2,5.2 \mathrm{~Hz}, 1 \mathrm{H}), 1.50-1.40(\mathrm{~m}, 3 \mathrm{H}), 1.26-1.21(\mathrm{~m}, 1 \mathrm{H}), 1.19(\mathrm{~s}$, $3 \mathrm{H}), 1.08(\mathrm{dd}, J=12.2,2.3 \mathrm{~Hz}, 1 \mathrm{H}), 1.05-0.98(\mathrm{~m}, 1 \mathrm{H}), 0.96(\mathrm{~s}$, $3 \mathrm{H}), 0.92(\mathrm{~s}, 3 \mathrm{H}), 0.89(\mathrm{~s}, 3 \mathrm{H}) ;{ }^{13} \mathrm{C}\left\{{ }^{1} \mathrm{H}\right\} \operatorname{NMR}\left(100 \mathrm{MHz}, \mathrm{CDCl}_{3}\right) \delta$ 175.3, 163.9, 158.8, 141.3, 112.6, 108.1, 104.9, 78.4, 56.1, 51.5, 41.8, $40.8,39.2,37.0,33.4,33.2,24.1,21.6,20.8,19.7,18.5,16.7,14.9)$; ${ }^{13} \mathrm{C}\left\{{ }^{1} \mathrm{H}\right\}$ NMR $\left(126 \mathrm{MHz}, \mathrm{CD}_{3} \mathrm{OD}\right) \delta 175.6,164.5,158.9,141.7$, 112.8 , 108.8, 104.9, 79.0, 57.5, 53.2, 43.0, 42.1, 40.4, 38.1, 34.2, 33.9, 24.2, 22.0, 21.1, 20.8, 19.6, 17.7, 15.4; IR $\nu_{\max }$ (neat) 2927, 1621, $1574,1454,1378,1262,1126 \mathrm{~cm}^{-1}$; HRMS (ESI) $\mathrm{m} / z[\mathrm{M}+\mathrm{H}]^{+}$ calcd for $\mathrm{C}_{23} \mathrm{H}_{33} \mathrm{O}_{4}$ 373.2383, found 373.2379. Anal. Calcd for $\mathrm{C}_{23} \mathrm{H}_{32} \mathrm{O}_{4}$ : C, 74.16; $\mathrm{H}, 8.66$. Found: $\mathrm{C}, 74.22 ; \mathrm{H}, 8.78$.

7-(Allyloxy)-2,2,5-trimethyl-8-((2E,6E)-3,7,11-trimethyldodeca2,6,10-trien-1-yl)-4H-benzo[d][1,3]dioxin-4-one (28). Allyl bromide $(0.65 \mathrm{~mL}, 7.50 \mathrm{mmol})$ was added with stirring to a suspension of $\mathrm{K}_{2} \mathrm{CO}_{3}(1.38 \mathrm{~g}, 10.0 \mathrm{mmol})$ and resorcylate $14(2.06 \mathrm{~g}, 5.00 \mathrm{mmol})$ in $\mathrm{Me}_{2} \mathrm{CO}(50 \mathrm{~mL})$. The resulting suspension was heated to $60^{\circ} \mathrm{C}$ with stirring for $18 \mathrm{~h}$, when the mixture was concentrated and diluted with $\mathrm{H}_{2} \mathrm{O}(50 \mathrm{~mL})$ and $\mathrm{CH}_{2} \mathrm{Cl}_{2}(50 \mathrm{~mL})$. The two phases were separated, and the aqueous layer was extracted with $\mathrm{CH}_{2} \mathrm{Cl}_{2}(3 \times 50 \mathrm{~mL})$. The combined organic layers were dried $\left(\mathrm{MgSO}_{4}\right)$, filtered, concentrated, and chromatographed (pentane/ $\mathrm{Et}_{2} \mathrm{O} 9: 1$ ) to provide allyl ether 28 $\left(1.61 \mathrm{~g}, 3.56 \mathrm{mmol}, 71 \%\right.$ ) as a pale yellow oil: $R_{f} 0.33$ (pentane $/ \mathrm{Et}_{2} \mathrm{O}$ 9:1); ${ }^{1} \mathrm{H}$ NMR $\left(400 \mathrm{MHz}, \mathrm{CDCl}_{3}\right) \delta 6.41(\mathrm{~s}, 1 \mathrm{H}), 6.04$ (ddt, $J=17.3$, $10.3,5.1 \mathrm{~Hz}, 1 \mathrm{H}), 5.42(\mathrm{dd}, J=17.3,1.6 \mathrm{~Hz}, 1 \mathrm{H}), 5.30(\mathrm{dd}, J=10.6$, $1.4 \mathrm{~Hz}, 1 \mathrm{H}), 5.13(\mathrm{tq}, J=7.3,1.3 \mathrm{~Hz}, 1 \mathrm{H}), 5.07(\mathrm{tdd}, J=6.9,3.1,1.4$ $\mathrm{Hz}, 2 \mathrm{H}), 4.60(\mathrm{dt}, J=5.1,1.6 \mathrm{~Hz}, 2 \mathrm{H}), 3.29(\mathrm{~d}, J=7.3 \mathrm{~Hz}, 2 \mathrm{H}), 2.63$ $(\mathrm{s}, 3 \mathrm{H}), 2.10-1.89(\mathrm{~m}, 8 \mathrm{H}), 1.75(\mathrm{~s}, 3 \mathrm{H}), 1.68-1.66(\mathrm{~m}, 9 \mathrm{H}), 1.58$ $(\mathrm{s}, 3 \mathrm{H}), 1.56(\mathrm{~s}, 3 \mathrm{H}) ;{ }^{13} \mathrm{C}\left\{{ }^{1} \mathrm{H}\right\}$ NMR $\left(100 \mathrm{MHz}, \mathrm{CDCl}_{3}\right) \delta 160.9$, $155.6,142.5,135.2$, 134.9, 132.6, 131.2, 124.3, 124.1, 121.6, 117.6, $116.0,109.3,105.7,104.7,68.9,39.74,39.66,26.7,26.5,25.73,25.66$, 22.5, 21.8, 17.6, 16.1, 16.0; IR $\nu_{\max }$ (neat) 2969, 3925, 2856, 1729, $1606,1575,1376,1278,1207,1169,1115,1046,980,908 \mathrm{~cm}^{-1}$; HRMS (ESI) $m / z[\mathrm{M}+\mathrm{H}]^{+}$calcd for $\mathrm{C}_{29} \mathrm{H}_{41} \mathrm{O}_{4} 453.3005$, found 453.2983. Anal. Calcd for $\mathrm{C}_{29} \mathrm{H}_{40} \mathrm{O}_{4}$ : C, 76.95; H, 8.91. Found: C, 76.84; H, 8.87.

7-(Allyloxy)-8-((R,2E,6E)-10,11-dihydroxy-3,7,11-trimethyldodeca-2,6-dien-1-yl)-2,2,5-trimethyl-4H-benzo[d][1,3]dioxin-4-one (30). Allyl ether $28(1.53 \mathrm{~g}, 3.38 \mathrm{mmol})$ was dissolved in ${ }^{t} \mathrm{BuOH}(17$ $\mathrm{mL})$ and $\mathrm{H}_{2} \mathrm{O}(17 \mathrm{~mL})$ and cooled to $0{ }^{\circ} \mathrm{C}$. Ligand $29(35 \mathrm{mg}, 33.8$ $\mu \mathrm{mol}), \mathrm{MeSO}_{4} \mathrm{NH}_{2}(322 \mathrm{mg}, 3.39 \mathrm{mmol}), \mathrm{K}_{3} \mathrm{Fe}(\mathrm{CN})_{6}(3.34 \mathrm{~g}, 10.1$ $\mathrm{mmol}), \mathrm{K}_{2} \mathrm{CO}_{3}(1.40 \mathrm{~g}, 10.1 \mathrm{mmol})$, and $\mathrm{K}_{2} \mathrm{OsO}_{4} \cdot 2 \mathrm{H}_{2} \mathrm{O}(6.3 \mathrm{mg}$, $17.1 \mu \mathrm{mol}$ ) were added sequentially with stirring at $0{ }^{\circ} \mathrm{C}$. After $24 \mathrm{~h}$, solid $\mathrm{Na}_{2} \mathrm{SO}_{3}(3.00 \mathrm{~g})$ was added, and the mixture was further stirred for $30 \mathrm{~min}$. The reaction mixture was diluted with $\mathrm{H}_{2} \mathrm{O}(25 \mathrm{~mL})$ and EtOAc $(25 \mathrm{~mL})$, the two phases were separated, and the aqueous layer was extracted with EtOAc $(3 \times 25 \mathrm{~mL})$. The combined organic layers were dried $\left(\mathrm{MgSO}_{4}\right)$, filtered, concentrated, and chromatographed (pentane/EtOAc 4:1-1:1 to EtOAc/EtOH 4:1) to afford unreacted allyl ether 28 (400 mg, $0.88 \mathrm{mmol}, 26 \%)$, tetraol (204 mg, $0.392 \mathrm{mmol}, 12 \%$ ), ligand 29 (33 mg, $32.3 \mu \mathrm{mol}, 96 \%$ recovered), and diol 30 (956 mg, $1.96 \mathrm{mmol}, 58 \%, 78 \%$ corrected for recovered allyl ether $28,88 \%$ ee as determined by Mosher ester analysis $)^{15}$ as a colorless oil: $R_{f} 0.36$ (pentane/EtOAc 1:1); $[\alpha]_{\mathrm{D}}^{23}+10.4$ (c 1.0, $\left.\mathrm{CHCl}_{3}\right) ;{ }^{1} \mathrm{H} \mathrm{NMR}\left(400 \mathrm{MHz}, \mathrm{CDCl}_{3}\right) \delta 6.42(\mathrm{~s}, 1 \mathrm{H}), 6.05$ (ddt, $J=$ $17.3,10.4,5.1 \mathrm{~Hz}, 1 \mathrm{H}), 5.42(\mathrm{dd}, J=17.3,1.6 \mathrm{~Hz}, 1 \mathrm{H}), 5.30(\mathrm{dd}, J=$ $10.6,1.4 \mathrm{~Hz}, 1 \mathrm{H}), 5.19-5.10(\mathrm{~m}, 2 \mathrm{H}), 4.61(\mathrm{dt}, J=5.1,1.6 \mathrm{~Hz}, 2 \mathrm{H})$, $3.33(\mathrm{dd}, J=10.5,2.0 \mathrm{~Hz}, 1 \mathrm{H}), 3.29(\mathrm{~d}, J=7.4 \mathrm{~Hz}, 2 \mathrm{H}), 2.63(\mathrm{~s}, 3 \mathrm{H})$, $2.25-1.92(\mathrm{~m}, 6 \mathrm{H}), 1.75(\mathrm{~s}, 3 \mathrm{H}), 1.71(\mathrm{~s}, 2 \mathrm{H}), 1.67(\mathrm{~s}, 6 \mathrm{H}), 1.59(\mathrm{~s}$, $3 \mathrm{H}), 1.56-1.45(\mathrm{~m}, 1 \mathrm{H}), 1.46-1.32(\mathrm{~m}, 1 \mathrm{H}), 1.18(\mathrm{~s}, 3 \mathrm{H}), 1.14(\mathrm{~s}$, $3 \mathrm{H}) ;{ }^{13} \mathrm{C}\left\{{ }^{1} \mathrm{H}\right\}$ NMR $\left(100 \mathrm{MHz}, \mathrm{CDCl}_{3}\right) \delta 161.14,161.08,155.7$, 
$142.7,135.2,135.1,132.8,125.1,121.9,117.9,116.1,109.5,105.9$, 104.9, 78.4, 73.1, 69.1, 39.9, 36.9, 29.8, 26.7, 26.6, 25.9 (2C), 23.4, 22.6, 21.9, 16.2, 16.0; IR $\nu_{\max }$ (neat) 3443, 2931, 2972, 1729, $1606,1576,1451,1377,1329,1281,1209,1170,1117 \mathrm{~cm}^{-1}$; HRMS (ESI) $m / z[\mathrm{M}+\mathrm{H}]^{+}$calcd for $\mathrm{C}_{29} \mathrm{H}_{43} \mathrm{O}_{6}$ 487.3060, found 487.3051. Anal. Calcd for $\mathrm{C}_{29} \mathrm{H}_{42} \mathrm{O}_{6}$ : C, 71.57; H, 8.70. Found: C, 71.69; H, 8.73.

7-(Allyloxy)-8-((2E,6E)-9-((S)-3,3-dimethyloxiran-2-yl)-3,7-dimethylnona-2,6-dien-1-yl)-2,2,5-trimethyl-4H-benzo[d][1,3]dioxin-4one (31). Pyridine (2.20 mL, $27.3 \mathrm{mmol})$ and $\mathrm{MsCl}(0.28 \mathrm{~mL}, 3.62$ mmol) were added sequentially with stirring to diol 30 in $\mathrm{CH}_{2} \mathrm{Cl}_{2}(20$ $\mathrm{mL})$. After $15 \mathrm{~h}$, the mixture was diluted with $\mathrm{Me}_{2} \mathrm{CO}(50 \mathrm{~mL})$, $\mathrm{K}_{2} \mathrm{CO}_{3}(20.0 \mathrm{~g}, 0.145 \mathrm{~mol})$ was added, and stirring was continued for 24 h. $\mathrm{H}_{2} \mathrm{O}(30 \mathrm{~mL})$ was added, and the two phases were separated. The aqueous layer was extracted with $\mathrm{CH}_{2} \mathrm{Cl}_{2}(3 \times 40 \mathrm{~mL})$, and the combined organic layers were dried $\left(\mathrm{MgSO}_{4}\right)$, filtered, concentrated, and chromatographed (pentane/EtOAc 19:1 to 5:1) to give epoxide $31(767 \mathrm{mg}, 1.64 \mathrm{mmol}, 90 \%)$ as a colorless oil: $R_{f} 0.25$ (pentane/ EtOAc 19:1); $[\alpha]_{\mathrm{D}}^{25}-1.8\left(\right.$ c 1.0, $\left.\mathrm{CHCl}_{3}\right) ;{ }^{1} \mathrm{H}$ NMR $(400 \mathrm{MHz}$, $\left.\mathrm{CDCl}_{3}\right) \delta 6.41(\mathrm{~s}, 1 \mathrm{H}), 6.16-5.90(\mathrm{~m}, 1 \mathrm{H}), 5.46-5.36(\mathrm{~m}, 1 \mathrm{H})$, $5.32-5.26(\mathrm{~m}, 1 \mathrm{H}), 5.17-5.05(\mathrm{~m}, 2 \mathrm{H}), 4.60(\mathrm{~d}, J=5.0 \mathrm{~Hz}, 2 \mathrm{H})$, $3.28(\mathrm{~d}, J=7.3 \mathrm{~Hz}, 2 \mathrm{H}), 2.66(\mathrm{t}, J=6.3 \mathrm{~Hz}, 1 \mathrm{H}), 2.62(\mathrm{~s}, 3 \mathrm{H}), 2.17-$ $1.91(\mathrm{~m}, 6 \mathrm{H}), 1.74(\mathrm{~s}, 3 \mathrm{H}), 1.66(\mathrm{~s}, 6 \mathrm{H}), 1.57(\mathrm{~s}, 3 \mathrm{H}), 1.67-1.48(\mathrm{~m}$, $2 \mathrm{H}), 1.28(\mathrm{~s}, 3 \mathrm{H}), 1.23(\mathrm{~s}, 3 \mathrm{H}) ;{ }^{13} \mathrm{C}\left\{{ }^{1} \mathrm{H}\right\} \operatorname{NMR}\left(100 \mathrm{MHz}, \mathrm{CDCl}_{3}\right) \delta$ 160.9 (2C), 155.5, 142.5, 135.0, 134.0, 132.6, 124.7, 121.7, 117.6, $115.9,109.3,105.7,104.6,68.9,64.1,58.2,39.7,36.2,27.4,26.5,25.7$ (2C), 24.9, 22.4, 21.8, 18.7, 16.1, 15.9; IR $\nu_{\max }$ (neat) 2961, 2923, $1727,1606,1575,1450,1376,1327,1279,1207,1169,1115 \mathrm{~cm}^{-1}$; HRMS (ESI) $m / z[M+H]^{+}$calcd for $\mathrm{C}_{29} \mathrm{H}_{41} \mathrm{O}_{5}$ 469.2954, found 469.2960 .

8-((2E,6E)-9-((S)-3,3-Dimethyloxiran-2-yl)-3,7-dimethylnona-2,6dien-1-yl)-7-hydroxy-2,2,5-trimethyl-4H-benzo[d][1,3]dioxin-4-one (33). Dimethylbarbituric acid (32) (217 $\mathrm{mg}, 1.39 \mathrm{mmol})$ and $\mathrm{Pd}\left(\mathrm{PPh}_{3}\right)_{4}(29 \mathrm{mg}, 0.0251 \mathrm{mmol})$ were added sequentially with stirring to epoxide $31(589 \mathrm{mg}, 1.26 \mathrm{mmol})$ in $\mathrm{CH}_{2} \mathrm{Cl}_{2}(10 \mathrm{~mL})$. After $1 \mathrm{~h}$, the reaction mixture was concentrated and purified by chromatography (pentane/EtOAc 4:1) to afford epoxide 33 (491 $\mathrm{mg}, 1.15 \mathrm{mmol}, 91 \%$ ) as a colorless oil: $R_{f} 0.30$ (pentane/EtOAc $4: 1$ ); $[\alpha]_{\mathrm{D}}^{26}+13.8\left(c 1.0, \mathrm{CHCl}_{3}\right) ;{ }^{1} \mathrm{H}$ NMR $\left(500 \mathrm{MHz}, \mathrm{CDCl}_{3}\right) \delta 6.90(\mathrm{~s}$, $1 \mathrm{H}), 6.42(\mathrm{~s}, 1 \mathrm{H}), 5.19-5.13(\mathrm{~m}, 1 \mathrm{H}), 5.12-5.05(\mathrm{~m}, 1 \mathrm{H}), 3.29(\mathrm{~d}, J$ $=7.3 \mathrm{~Hz}, 2 \mathrm{H}), 2.77(\mathrm{dd}, J=7.4,4.9 \mathrm{~Hz}, 1 \mathrm{H}), 2.58(\mathrm{~s}, 3 \mathrm{H}), 2.19-1.98$ $(\mathrm{m}, 6 \mathrm{H}), 1.74(\mathrm{~s}, 3 \mathrm{H}), 1.73-1.69(\mathrm{~m}, 1 \mathrm{H}), 1.68(\mathrm{~s}, 6 \mathrm{H}), 1.57(\mathrm{~s}, 3 \mathrm{H})$, $1.56-1.49(\mathrm{~m}, 1 \mathrm{H}), 1.33(\mathrm{~s}, 3 \mathrm{H}), 1.29(\mathrm{~s}, 3 \mathrm{H}) ;{ }^{13} \mathrm{C}\left\{{ }^{1} \mathrm{H}\right\}$ NMR $(100$ $\left.\mathrm{MHz} \mathrm{CDCl}_{3}\right) \delta 161.1,160.4,155.9,142.6,136.8,134.0,124.5,121.9$, $113.5,113.2,105.0,104.7,64.6,59.5,39.3,36.2,27.0,25.7,25.7$ (2C), $24.9,22.0,21.8,18.7,16.0$ (2C); IR $\nu_{\max }$ (neat) $3258,2964,2927$, $1727,1693,1609,1514,1452,1377,1327,1276,1210,1107 \mathrm{~cm}^{-1}$; HRMS (ESI) $m / z[\mathrm{M}+\mathrm{H}]^{+}$calcd for $\mathrm{C}_{26} \mathrm{H}_{37} \mathrm{O}_{5} 429.2641$, found 429.2645 .

(7aR,9aR,11S, 13aS, 13bR)-11-Hydroxy-2,2,5,7a, 10, 10,13a-heptamethyl-7a, 8,9a,10,11,12,13,13a,13b,14-decahydro-4H,9H-benzo[a][1,3]dioxino[5,4-j]xanthen-4-one (34). $\mathrm{FeCl}_{3} \cdot 6 \mathrm{H}_{2} \mathrm{O}(892 \mathrm{mg}, 3.30$ $\mathrm{mmol}$ ) was added with stirring to epoxide $33(470 \mathrm{mg}, 1.10 \mathrm{mmol})$ in $\mathrm{MeNO}_{2}(220 \mathrm{~mL})$, and the resulting mixture was further stirred at 25 ${ }^{\circ} \mathrm{C}$ for $15 \mathrm{~min}$. Saturated aqueous $\mathrm{NaHCO}_{3}(150 \mathrm{~mL})$ was added, and the two phases were separated. The aqueous layer was extracted with $\mathrm{CH}_{2} \mathrm{Cl}_{2}(3 \times 50 \mathrm{~mL})$, and the combined organic layers were dried $\left(\mathrm{MgSO}_{4}\right)$, filtered, concentrated, and chromatographed (pentane/ EtOAc 3:1) to give meroterpenoid $34(263 \mathrm{mg}, 0.614 \mathrm{mmol}, 56 \%$, $92 \%$ ee, measured by chiral HPLC, Chiralpak IE column, nhexane $/{ }^{i} \mathrm{PrOH} 17: 3,5 \mathrm{~mL} / \mathrm{min}, t_{\mathrm{R}}=18.9[(-)$-enantiomer], 25.8 $[(+)$-enantiomer $] \mathrm{min}$ ) as a white foam: $R_{f} 0.19$ (pentane/EtOAc $3: 1) ;[\alpha]_{\mathrm{D}}^{24}+52.2\left(c 1.0, \mathrm{CHCl}_{3}\right) ;{ }^{1} \mathrm{H}$ NMR $\left(400 \mathrm{MHz}^{-} \mathrm{CDCl}_{3}\right) \delta$ $6.33(\mathrm{~s}, 1 \mathrm{H}), 3.26(\mathrm{dt}, J=11.0,5.3 \mathrm{~Hz}, 1 \mathrm{H}), 2.56(\mathrm{~s}, 3 \mathrm{H}), 2.56-2.49$ $(\mathrm{m}, 1 \mathrm{H}), 2.25(\mathrm{dd}, J=16.7,13.1 \mathrm{~Hz}, 1 \mathrm{H}), 2.10(\mathrm{dt}, J=12.5,3.2 \mathrm{~Hz}$, $1 \mathrm{H}), 1.86-1.76(\mathrm{~m}, 2 \mathrm{H}), 1.72(\mathrm{~s}, 3 \mathrm{H}), 1.68(\mathrm{~s}, 3 \mathrm{H}), 1.73-1.60(\mathrm{~m}$, $3 \mathrm{H}), 1.52(\mathrm{dd}, J=13.1,5.0 \mathrm{~Hz}, 1 \mathrm{H}), 1.47-1.41(\mathrm{~m}, 1 \mathrm{H}), 1.40(\mathrm{~d}, J=$ $5.9 \mathrm{~Hz}, 1 \mathrm{H}), 1.19(\mathrm{~s}, 3 \mathrm{H}), 1.12(\mathrm{td}, J=13.0,4.3 \mathrm{~Hz}, 1 \mathrm{H}), 1.03(\mathrm{~s}$, $3 \mathrm{H}), 1.02-0.98(\mathrm{~m}, 1 \mathrm{H}), 0.92(\mathrm{~s}, 3 \mathrm{H}), 0.82(\mathrm{~s}, 3 \mathrm{H}) ;{ }^{13} \mathrm{C}\left\{{ }^{1} \mathrm{H}\right\} \mathrm{NMR}$ $\left(101 \mathrm{MHz}, \mathrm{CDCl}_{3}\right) \delta 160.8,158.6,156.1,142.1,114.3,108.0,104.8$,
104.0, 78.6, 78.1, 55.0, 51.1, 40.7, 38.8, 37.4, 36.7, 28.1, 27.1, 26.1, $25.5,22.0,20.7,19.4,16.5,15.5,15.0$; IR $\nu_{\max }$ (neat) 3440, 2930, $2867,1713,1616,1574,1452,1388,1286,1207,1126,1043 \mathrm{~cm}^{-1}$; HRMS (ESI) $m / z[M+\mathrm{H}]^{+}$calcd for $\mathrm{C}_{26} \mathrm{H}_{37} \mathrm{O}_{5} 429.2641$, found 429.2646.

(3S, 4aR,6aR, 12aR, 12bS)-3,11-Dihydroxy-4,4,6a,9,12b-pentamethyl-1,3,4,4a,5,6,6a, 12,12a,12b-decahydro-2H-benzo[a]xanthene10-carboxylic Acid (35). $\mathrm{H}_{2} \mathrm{O}(10 \mu \mathrm{L}, 0.552 \mathrm{mmol})$ was added to a suspension of $\mathrm{KO}^{t} \mathrm{Bu}(124 \mathrm{mg}, 1.10 \mathrm{mmol})$ in $\mathrm{Et}_{2} \mathrm{O}(1 \mathrm{~mL})$ at $0{ }^{\circ} \mathrm{C}$ and stirred for $5 \mathrm{~min}$. Meroterpenoid $34(59 \mathrm{mg}, 0.138 \mathrm{mmol})$ in $\mathrm{Et}_{2} \mathrm{O}(1 \mathrm{~mL})$ was added with stirring at $25{ }^{\circ} \mathrm{C}$. After $3 \mathrm{~h}$, ice was added until two layers were formed, and the two phases were separated and diluted with $\mathrm{Et}_{2} \mathrm{O}(2 \mathrm{~mL})$. The $\mathrm{pH}$ was adjusted to $\sim 1$ with aqueous $\mathrm{HCl}(4 \mathrm{M})$. The two phases were separated, and the aqueous layer was extracted with $\mathrm{Et}_{2} \mathrm{O}(3 \times 2 \mathrm{~mL})$. The combined organic layers were dried $\left(\mathrm{MgSO}_{4}\right)$, filtered, concentrated, and chromatographed (pentane/EtOAc/AcOH 3:1:0.01) to give the carboxylic acid $35(37 \mathrm{mg}, 0.0952 \mathrm{mmol}, 69 \%)$ as a white solid: $R_{\text {f }}$ 0.14 (pentane/EtOAc/AcOH 4:1:0.01); mp 153-155 ${ }^{\circ} \mathrm{C} ;[\alpha]_{\mathrm{D}}^{24}$ $+104.3\left(c 0.3, \mathrm{CH}_{3} \mathrm{OH}\right) ;{ }^{1} \mathrm{H}$ NMR $\left(400 \mathrm{MHz}, \mathrm{CD}_{3} \mathrm{OD}\right) \delta 6.08(\mathrm{~s}$, $1 \mathrm{H}), 3.18(\mathrm{dd}, J=11.3,5.0 \mathrm{~Hz}, 1 \mathrm{H}), 2.61(\mathrm{dd}, J=16.8,5.0 \mathrm{~Hz}, 1 \mathrm{H})$, $2.45(\mathrm{~s}, 3 \mathrm{H}), 2.26(\mathrm{dd}, J=16.7,13.0 \mathrm{~Hz}, 1 \mathrm{H}), 2.03(\mathrm{dt}, J=12.4,3.2$ $\mathrm{Hz}, 1 \mathrm{H}), 1.84-1.73(\mathrm{~m}, 2 \mathrm{H}), 1.72-1.59(\mathrm{~m}, 3 \mathrm{H}), 1.54-1.41(\mathrm{~m}$, $2 \mathrm{H}), 1.16(\mathrm{~s}, 3 \mathrm{H}), 1.15-1.08(\mathrm{~m}, 1 \mathrm{H}), 1.04-1.01(\mathrm{~m}, 1 \mathrm{H}), 1.00(\mathrm{~s}$, $3 \mathrm{H}), 0.94(\mathrm{~s}, 3 \mathrm{H}), 0.81(\mathrm{~s}, 3 \mathrm{H}) ;{ }^{13} \mathrm{C}\left\{{ }^{1} \mathrm{H}\right\}$ NMR $\left(101 \mathrm{MHz}, \mathrm{CD}_{3} \mathrm{OD}\right)$ $\delta 175.6,164.4,158.7,141.7,112.7,108.6,105.2,79.4,78.7,56.5,52.9$, 42.1, 39.9, 38.8, 37.8, 28.7, 27.9, 24.2, 21.0, 20.5, 17.8, 16.2, 15.5; IR $\nu_{\max }$ (neat) $3445,2972,2934,2865,1621,1579,1453,1379,1265$, 1178, 1126, $1038 \mathrm{~cm}^{-1}$; HRMS (ESI) $\mathrm{m} / z[\mathrm{M}-\mathrm{H}]^{-}$Calcd for $\mathrm{C}_{23} \mathrm{H}_{31} \mathrm{O}_{5}$ 387.2171, found 387.2180 .

(+)-Hongoquercin $B[(3 S, 4 a R, 6 a R, 12 a R, 12 b S)-3$-Acetoxy-11-hydroxy-4,4,6a,9,12b-pentamethyl-1,3,4,4a,5,6,6a,12,12a,12b-decahydro-2H-benzo[a]xanthene-10-carboxylic Acid (2)]. Ac $\mathrm{A}_{2} \mathrm{O}(63 \mu \mathrm{L}$, $0.666 \mathrm{mmol}$ ) was added with stirring to carboxylic acid $35(37 \mathrm{mg}$ $0.0952 \mathrm{mmol})$ in pyridine $(0.5 \mathrm{~mL})$ at $25^{\circ} \mathrm{C}$. After $24 \mathrm{~h}, \mathrm{CH}_{2} \mathrm{Cl}_{2}(2$ $\mathrm{mL}$ ) was added, and the $\mathrm{pH}$ was adjusted to $\sim 1$ with aqueous $\mathrm{HCl}$ (4 $\mathrm{M})$. The two phases were separated, and the aqueous layer was extracted with $\mathrm{CH}_{2} \mathrm{Cl}_{2}(3 \times 2 \mathrm{~mL})$. The combined organic layers were dried $\left(\mathrm{MgSO}_{4}\right)$, filtered, and concentrated to give diacetate 36 . Crude diacetate 36 was dissolved in $\mathrm{MeOH}(2 \mathrm{~mL})$ and $\mathrm{H}_{2} \mathrm{O}(0.2$ $\mathrm{mL})$, and $\mathrm{K}_{2} \mathrm{CO}_{3}(20 \mathrm{mg}, 0.143 \mathrm{mmol})$ was added at $25{ }^{\circ} \mathrm{C}$. The resulting mixture was stirred for $5 \mathrm{~h}$, when $\mathrm{CH}_{2} \mathrm{Cl}_{2}(2 \mathrm{~mL})$ was added, and the $\mathrm{pH}$ was adjusted to $\sim 1$ with aqueous $\mathrm{HCl}(4 \mathrm{M})$. The two phases were separated, and the aqueous layer was extracted with $\mathrm{CH}_{2} \mathrm{Cl}_{2}(3 \times 1 \mathrm{~mL})$. The combined organic layers were dried (MgSO4), filtered, concentrated, and chromatographed (pentane/ $\mathrm{EtOAc} / \mathrm{AcOH}$ 3:1:0.01) to give (+)-hongoquercin B (2) (27 mg, $0.0627 \mathrm{mmol}, 66 \%$ over two steps from carboxylic acid 35) as white solid: $R_{f} 0.29$ (pentane/EtOAc/AcOH 3:1:0.01); mp $155-157{ }^{\circ} \mathrm{C}$;

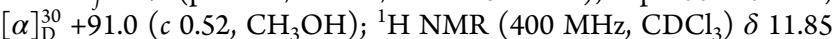
$(\mathrm{s}, 1 \mathrm{H}), 6.21(\mathrm{~s}, 1 \mathrm{H}), 4.52(\mathrm{dd}, J=11.6,4.7 \mathrm{~Hz}, 1 \mathrm{H}), 2.66(\mathrm{dd}, J=$ $16.8,4.8 \mathrm{~Hz}, 1 \mathrm{H}), 2.52(\mathrm{~s}, 3 \mathrm{H}), 2.30(\mathrm{dd}, J=16.8,13.1 \mathrm{~Hz}, 1 \mathrm{H})$, $2.13-2.07(\mathrm{~m}, 1 \mathrm{H}), 2.07(\mathrm{~s}, 3 \mathrm{H}), 1.86(\mathrm{dt}, J=13.3,3.6 \mathrm{~Hz}, 1 \mathrm{H})$, $1.81-1.60(\mathrm{~m}, 4 \mathrm{H}), 1.53(\mathrm{dd}, J=13.1,5.0 \mathrm{~Hz}, 1 \mathrm{H}), 1.47-1.42(\mathrm{~m}$, $1 \mathrm{H}), 1.24-1.18(\mathrm{~m}, 1 \mathrm{H}), 1.20(\mathrm{~s}, 3 \mathrm{H}), 1.10(\mathrm{dd}, J=12.1,2.2 \mathrm{~Hz}$, $1 \mathrm{H}), 0.96(\mathrm{~s}, 3 \mathrm{H}), 0.91(\mathrm{~s}, 3 \mathrm{H}), 0.90(\mathrm{~s}, 3 \mathrm{H}) ;{ }^{13} \mathrm{C}\left\{{ }^{1} \mathrm{H}\right\}$ NMR $(126$ $\left.\mathrm{MHz} \mathrm{CDCl}_{3}\right) \delta 175.9,171.0,163.8,158.6,141.6,112.6,107.7,102.7$ 80.4, 78.0, 55.1, 51.3, 40.6, 37.7, 37.1, 36.6, 28.1, 24.1, 23.5, 21.3, 20.7, 19.3, 16.8, 16.7, 15.0; IR $\nu_{\max }$ (neat) $3063,2972,2941,1731$, $1623,1580,1454,1371,1263,1178,1126,1035,1007 \mathrm{~cm}^{-1}$; HRMS (ESI) $m / z[\mathrm{M}-\mathrm{H}]^{-}$calcd for $\mathrm{C}_{25} \mathrm{H}_{33} \mathrm{O}_{6} 429.2277$, found 429.2284 .

(E)-7-(tert-Butyldimethylsilyloxy)-8-(3,7-dimethylocta-2,6-dienyl)-2,2,5-trimethyl-4H-benzo[d][1,3]dioxin-4-one (37). $\mathrm{NEt}_{3}$ (1.08 $\mathrm{mL}, 7.72 \mathrm{mmol}$ ) was added with stirring to resorcylate $24(806 \mathrm{mg}$, $2.34 \mathrm{mmol})$ in $\mathrm{CH}_{2} \mathrm{Cl}_{2}(50 \mathrm{~mL})$ at $25^{\circ} \mathrm{C}$, when ${ }^{t} \mathrm{BuMe}_{2} \mathrm{SiCl}(1.16 \mathrm{~g}$, $7.72 \mathrm{mmol})$ and DMAP $(5.72 \mathrm{mg}, 0.0468 \mathrm{mmol})$ were added. The resulting mixture was stirred for $2 \mathrm{~h}$, when $10 \%$ aqueous citric acid $(30 \mathrm{~mL})$ and $\mathrm{CH}_{2} \mathrm{Cl}_{2}(20 \mathrm{~mL})$ were added. The two phases were separated, and the organic layer was washed with $10 \%$ citric acid solution $(2 \times 30 \mathrm{~mL}), \mathrm{H}_{2} \mathrm{O}(30 \mathrm{~mL})$, and brine $(30 \mathrm{~mL})$. The organic 
layer was dried $\left(\mathrm{MgSO}_{4}\right)$, filtered, concentrated, and chromatographed (pentane/EtOAc 19:1) to give silyl ether 37 (815 mg, 1.78 mmol, $76 \%$ ) as a colorless oil: $R_{f} 0.59$ (pentane/ $\left.\mathrm{Et}_{2} \mathrm{O} 10: 1\right) ;{ }^{1} \mathrm{H}$ NMR $\left(400 \mathrm{MHz}, \mathrm{CDCl}_{3}\right) \delta 6.36(\mathrm{~s}, 1 \mathrm{H}), 5.13-5.00(\mathrm{~m}, 2 \mathrm{H}), 3.24(\mathrm{~d}, J=$ $7.0 \mathrm{~Hz}, 2 \mathrm{H}), 2.58(\mathrm{~s}, 3 \mathrm{H}), 2.07-1.99(\mathrm{~m}, 2 \mathrm{H}), 1.98-1.90(\mathrm{~m}, 2 \mathrm{H})$, $1.73(\mathrm{~s}, 3 \mathrm{H}), 1.65(\mathrm{~s}, 6 \mathrm{H}), 1.63(\mathrm{~s}, 3 \mathrm{H}), 1.55(\mathrm{~s}, 3 \mathrm{H}), 1.01(\mathrm{~s}, 9 \mathrm{H})$, $0.28(\mathrm{~s}, 6 \mathrm{H}) ;{ }^{13} \mathrm{C}\left\{{ }^{1} \mathrm{H}\right\}$ NMR $\left(100 \mathrm{MHz}, \mathrm{CDCl}_{3}\right) \delta 160.9,158.9$, $156.4,141.7,134.9,131.3,124.1,121.8,118.4,116.1,105.9,104.6$, $39.6,26.5,25.7$ (2C), 25.6 (4C), 22.2, 22.1, 18.2, 17.6, 16.2, -4.1 (2C); IR $\nu_{\max }$ (neat) 2930, 2859, 1733, 1606, 1569, 1292, 1209, 1169, 1044, 841, $782 \mathrm{~cm}^{-1}$; HRMS (ESI) $\mathrm{m} / z[\mathrm{M}+\mathrm{H}]^{+}$calcd for $\mathrm{C}_{27} \mathrm{H}_{43} \mathrm{O}_{4} \mathrm{Si}$ 459.2931, found 459.2938. Anal. Calcd for $\mathrm{C}_{27} \mathrm{H}_{42} \mathrm{O}_{4} \mathrm{Si}$ : C, 70.70; H, 9.23. Found: C, 70.52; H, 9.10.

(S,E)-7-((tert-Butyldimethylsilyl)oxy)-8-(5-(3,3-dimethyloxiran-2yl)-3-methylpent-2-en-1-yl)-2,2,5-trimethyl-4H-benzo[d] [1,3]dioxin-4-one (39). Silyl ether 37 (334 mg, $0.75 \mathrm{mmol}$ ) and the chiral ketone $38(58 \mathrm{mg}, 0.225 \mathrm{mmol})$ were dissolved in $\mathrm{CH}_{2} \mathrm{Cl}_{2}(6 \mathrm{~mL})$, $\mathrm{CH}_{3} \mathrm{CN}(3 \mathrm{~mL}), \mathrm{EtOH}(3 \mathrm{~mL})$, and aqueous buffer $\left(2 \mathrm{M} \mathrm{K}_{2} \mathrm{CO}_{3} ; 4\right.$ $\times 10^{-3}$ M EDTA; $\left.6 \mathrm{~mL}\right)$ and cooled to $0{ }^{\circ} \mathrm{C} . \mathrm{H}_{2} \mathrm{O}_{2}(0.42 \mathrm{~mL})$ was added dropwise with stirring. After $15 \mathrm{~h}, \mathrm{Na}_{2} \mathrm{SO}_{3}(200 \mathrm{mg})$ was added, and the phases were separated. The aqueous layer was extracted with $\mathrm{Et}_{2} \mathrm{O}(3 \times 15 \mathrm{~mL})$, and the combined organic layers were dried $\left(\mathrm{MgSO}_{4}\right)$, filtered, concentrated, and chromatographed (pentane/EtOAc 10:1) to afford epoxide $39(244 \mathrm{mg}, 0.514 \mathrm{mmol}$, $69 \%)$ as a colorless oil: $R_{f} 0.22$ (pentane/EtOAc 19:1); $[\alpha]_{\mathrm{D}}^{23}+2.8(c$ 1.0, $\left.\mathrm{CHCl}_{3}\right) ;{ }^{1} \mathrm{H} \mathrm{NMR}\left(400 \mathrm{MHz}, \mathrm{CDCl}_{3}\right) \delta 6.35(\mathrm{~s}, 1 \mathrm{H}), 5.12(\mathrm{tq}, J$ $=7.1,1.3 \mathrm{~Hz}, 1 \mathrm{H}), 3.24(\mathrm{~d}, J=7.0 \mathrm{~Hz}, 2 \mathrm{H}), 2.65(\mathrm{t}, J=6.2 \mathrm{~Hz}, 1 \mathrm{H})$, $2.58(\mathrm{~s}, 3 \mathrm{H}), 2.20-1.98(\mathrm{~m}, 2 \mathrm{H}), 1.74(\mathrm{~s}, 3 \mathrm{H}), 1.65(\mathrm{~s}, 6 \mathrm{H}), 1.61-$ $1.53(\mathrm{~m}, 2 \mathrm{H}), 1.24(\mathrm{~s}, 3 \mathrm{H}), 1.21(\mathrm{~s}, 3 \mathrm{H}), 1.00(\mathrm{~s}, 9 \mathrm{H}), 0.28(\mathrm{~s}, 6 \mathrm{H})$; ${ }^{13} \mathrm{C}\left\{{ }^{1} \mathrm{H}\right\}$ NMR $\left(100 \mathrm{MHz}, \mathrm{CDCl}_{3}\right) \delta 160.8,158.9,156.3,141.9$, 134.1, 122.5, 118.1, 116.1, 105.9, 104.6, 64.0, 58.2, 36.2, 27.3, 25.8 (2C), 25.6 (3C), 24.8, 22.2, 22.1, 18.6, 18.3, 16.2, -4.0 (2C); IR $\nu_{\max }$ (neat) 2959, 2930, 2859, 1732, 1605, 1570,1279, $842 \mathrm{~cm}^{-1}$; HRMS (FTMS + p APCI) $m / z[\mathrm{M}+\mathrm{H}]^{+}$calcd for $\mathrm{C}_{27} \mathrm{H}_{43} \mathrm{O}_{5} \mathrm{Si} 475.2874$, found 475.2870. Anal. Calcd for $\mathrm{C}_{27} \mathrm{H}_{42} \mathrm{O}_{5} \mathrm{Si}: \mathrm{C}, 68.31 ; \mathrm{H}, 8.92$. Found: C, 68.21; H, 9.03 .

$(S, E)-8$-(5-(3,3-Dimethyloxiran-2-yl)-3-methylpent-2-en-1-yl)-7hydroxy-2,2,5-trimethyl-4H-benzo[d][1,3]dioxin-4-one (40). $\mathrm{K}_{2} \mathrm{CO}_{3}$ $(2.33 \mathrm{~g}, 16.9 \mathrm{mmol})$ was added with stirring to epoxide $39(160 \mathrm{mg}$, $0.337 \mathrm{mmol})$ in $\mathrm{Me}_{2} \mathrm{CO}(20 \mathrm{~mL})$ at $25^{\circ} \mathrm{C}$. After $2 \mathrm{~h}, \mathrm{CH}_{2} \mathrm{Cl}_{2}(30$ $\mathrm{mL})$ and $\mathrm{H}_{2} \mathrm{O}(50 \mathrm{~mL})$ were added, and the two phases were separated. The aqueous layer was extracted with $\mathrm{CH}_{2} \mathrm{Cl}_{2}(3 \times 20$ $\mathrm{mL})$, and the combined organic layers were dried $\left(\mathrm{MgSO}_{4}\right)$, filtered, concentrated, and chromatographed (pentane/EtOAc 5:1) to give epoxide $40(112 \mathrm{mg}, 0.311 \mathrm{mmol}, 92 \%)$ as a colorless oil: $R_{f} 0.29$ (pentane/EtOAc 5:1); $[\alpha]_{\mathrm{D}}^{24}-9.4\left(\right.$ c $\left.1.0, \mathrm{CHCl}_{3}\right) ;{ }^{1} \mathrm{H}$ NMR $(400$ $\left.\mathrm{MHz}, \mathrm{CDCl}_{3}\right) \delta 6.40(\mathrm{~s}, 1 \mathrm{H}), 6.17(\mathrm{~s}, 1 \mathrm{H}), 5.30-5.15(\mathrm{~m}, 1 \mathrm{H}), 3.31$ $(\mathrm{dd}, J=7.0,2.1 \mathrm{~Hz}, 2 \mathrm{H}), 2.69(\mathrm{dd}, J=6.8,5.6 \mathrm{~Hz}, 1 \mathrm{H}), 2.58(\mathrm{~s}, 3 \mathrm{H})$, $2.26-2.07(\mathrm{~m}, 2 \mathrm{H}), 1.80(\mathrm{~s}, 3 \mathrm{H}), 1.68(\mathrm{~s}, 6 \mathrm{H}), 1.69-1.55(\mathrm{~m}, 2 \mathrm{H})$, 1.28 (s, 3H), 1.25 (s, 3H); ${ }^{13} \mathrm{C}\left\{{ }^{1} \mathrm{H}\right\}$ NMR $\left(100 \mathrm{MHz} \mathrm{CDCl}_{3}\right) \delta$ $160.9,159.8,156.0,142.8,136.8,121.7,113.6,113.0,105.4,104.8$, 64.2, 58.6, 36.5, 27.2, 25.8, 25.7, 24.8, 22.0, 21.8, 18.7, 16.2; IR $\nu_{\max }$ (neat) $3235,2928,1727,1696,1609,1277 \mathrm{~cm}^{-1}$; HRMS (FTMS + p APCI) $m / z[\mathrm{M}+\mathrm{H}]^{+}$calcd for $\mathrm{C}_{21} \mathrm{H}_{29} \mathrm{O}_{5} 361.2010$, found 361.2016.

(7aS, 10S, 11aS)-10-Hydroxy-2,2,5,7a, 11,11-hexamethyl-7a,8,10,11,11a,12-hexahydro-4H,9H-[1,3]dioxino[4,5-a]xanthen-4-one (41). $\mathrm{BF}_{3} \mathrm{OEt}_{2}(0.2 \mathrm{~mL}, 0.139 \mathrm{mmol})$ was added with stirring to epoxide $40(110 \mathrm{mg}, 0.305 \mathrm{mmol})$ in $\mathrm{CH}_{2} \mathrm{Cl}_{2}(30 \mathrm{~mL})$ at $-78{ }^{\circ} \mathrm{C}$. After $5 \mathrm{~min}, \mathrm{Et}_{3} \mathrm{~N}(5 \mathrm{~mL})$ and $\mathrm{H}_{2} \mathrm{O}(20 \mathrm{~mL})$ were added, the mixture was allowed to warm to $25{ }^{\circ} \mathrm{C}$, and the two phases were separated. The aqueous layer was extracted with $\mathrm{CH}_{2} \mathrm{Cl}_{2}(3 \times 30 \mathrm{~mL})$, and the combined organic layers were dried $\left(\mathrm{MgSO}_{4}\right)$, filtered, concentrated, and purified by chromatography (pentane/EtOAc 5:2) to give the meroterpenoid $41(85 \mathrm{mg}, 0.236 \mathrm{mmol}, 77 \%, 84 \%$ ee, measured by chiral HPLC, Chiralpak IE column, $n$-hexane $/{ }^{i} \mathrm{PrOH}$ 9:1, $5 \mathrm{~mL} / \mathrm{min}$, $t_{\mathrm{R}}=22.0[(-)$-enantiomer $], 26.6[(+)$-enantiomer $\left.] \mathrm{min}\right)$ as a white foam: $R_{f} 0.29$ (pentane/EtOAc 5:2); $[\alpha]_{\mathrm{D}}^{24}-71.0\left(\right.$ c 1.0, $\left.\mathrm{CHCl}_{3}\right) ;{ }^{1} \mathrm{H}$ NMR $\left(400 \mathrm{MHz}, \mathrm{CDCl}_{3}\right) \delta 6.33(\mathrm{~s}, 1 \mathrm{H}), 3.41(\mathrm{dd}, J=11.4,4.2 \mathrm{~Hz}$, $1 \mathrm{H}), 2.63(\mathrm{dd}, J=16.8,4.9 \mathrm{~Hz}, 1 \mathrm{H}), 2.56(\mathrm{~s}, 3 \mathrm{H}), 2.31(\mathrm{dd}, J=16.9$, $13.2 \mathrm{~Hz}, 1 \mathrm{H}), 2.00(\mathrm{dt}, J=12.5,3.3 \mathrm{~Hz}, 1 \mathrm{H}), 1.91-1.81(\mathrm{~m}, 1 \mathrm{H})$, $1.78-1.74(\mathrm{~m}, 1 \mathrm{H}), 1.73(\mathrm{~s}, 3 \mathrm{H}), 1.69(\mathrm{~s}, 3 \mathrm{H}), 1.64-1.52(\mathrm{~m}, 2 \mathrm{H})$, $1.20(\mathrm{~s}, 3 \mathrm{H}), 1.12(\mathrm{~s}, 3 \mathrm{H}), 0.89(\mathrm{~s}, 3 \mathrm{H}) ;{ }^{13} \mathrm{C}\left\{{ }^{1} \mathrm{H}\right\} \mathrm{NMR}(100 \mathrm{MHz}$, $\left.\mathrm{CDCl}_{3}\right) \delta 160.8,158.6,156.0,142.2,114.3,107.9,104.8,104.1,77.8$, 77.6, 46.1, 38.5, 37.4, 28.1, 27.3, 26.1, 25.4, 21.9, 19.8, 17.2, 14.3; IR $\nu_{\max }$ (neat) 3433, 2942, 1708, 1617, 1573, 1287, $1128 \mathrm{~cm}^{-1}$; HRMS (ESI) $m / z[\mathrm{M}-\mathrm{H}]^{-}$calcd for $\mathrm{C}_{21} \mathrm{H}_{27} \mathrm{O}_{5} 359.1858$, found 359.1853 . Anal. Calcd for $\mathrm{C}_{21} \mathrm{H}_{28} \mathrm{O}_{5}$ : C, 69.98; H, 7.83. Found: C, 69.86; H, 7.94.

General Procedure for BDSB (46)-Induced Halocyclization. BDSB (46) (604 mg, $1.10 \mathrm{mmol}$ ) was added with stirring to resorcylate 24 or $14(1.00 \mathrm{mmol})$ in $\mathrm{MeNO}_{2}(50 \mathrm{~mL})$ at $-25^{\circ} \mathrm{C}$. After $10 \mathrm{~min}$, saturated aqueous $\mathrm{NaHCO}_{3}(15 \mathrm{~mL})$ and aqueous $\mathrm{Na}_{2} \mathrm{SO}_{3}(0.5 \mathrm{M} ; 5 \mathrm{~mL})$ were added, and stirring was continued for 15 $\mathrm{min}$. The two phases were separated, and the aqueous layer was extracted with $\mathrm{CH}_{2} \mathrm{Cl}_{2}(3 \times 10 \mathrm{~mL})$. The combined organic layers were dried $\left(\mathrm{MgSO}_{4}\right)$, filtered, concentrated, and chromatographed (pentane $/ \mathrm{Et}_{2} \mathrm{O} 9: 1$ ) to give the bromo-meroterpenoid $\mathbf{4 2}$ or $\mathbf{4 4}$.

10-Bromo-2,2,5,7a, 11,11-hexamethyl-7a,8,10,11,11a,12-hexahydro-4H,9H-[1,3]dioxino[4,5-a]xanthen-4-one (42). Bromide 42 $(272 \mathrm{mg}, 0.642 \mathrm{mmol}, 64 \%)$, prepared from resorcylate 24 (344 mg, $1.00 \mathrm{mmol}$ ), was obtained as a white solid: $R_{f} 0.29$ (pentane/ $/ \mathrm{Et}_{2} \mathrm{O}$ 9:1); mp 225.3-225.7 ${ }^{\circ} \mathrm{C}$; ${ }^{1} \mathrm{H}$ NMR $\left(400 \mathrm{MHz},\left(\mathrm{CD}_{3}\right)_{2} \mathrm{CO}\right) \delta 6.36$ $(\mathrm{s}, 1 \mathrm{H}), 4.26(\mathrm{dd}, J=11.8,4.8 \mathrm{~Hz}, 1 \mathrm{H}), 2.80-2.77(\mathrm{~m}, 1 \mathrm{H}), 2.52(\mathrm{~s}$, $3 \mathrm{H}), 2.50-2.43(\mathrm{~m}, 1 \mathrm{H}), 2.30-2.13(\mathrm{~m}, 2 \mathrm{H}), 2.00(\mathrm{dt}, J=13.0,3.5$ $\mathrm{Hz}, 1 \mathrm{H}), 1.90-1.79(\mathrm{~m}, 2 \mathrm{H}), 1.70(\mathrm{~s}, 3 \mathrm{H}), 1.67(\mathrm{~s}, 3 \mathrm{H}), 1.27(\mathrm{~s}, 3 \mathrm{H})$, $1.19(\mathrm{~s}, 3 \mathrm{H}), 1.06(\mathrm{~s}, 3 \mathrm{H}) ;{ }^{13} \mathrm{C}\left\{{ }^{1} \mathrm{H}\right\}$ NMR $\left(101 \mathrm{MHz},\left(\mathrm{CD}_{3}\right)_{2} \mathrm{CO}\right) \delta$ 160.7, 159.1, 157.0, 142.6, 115.1, 109.3, 105.8, 105.4, 78.2, 66.9, 47.9, $41.0,40.1,32.3,29.9,26.3,25.6,22.1,20.2,19.5,17.4$; IR $\nu_{\max }$ (neat) $2975,2924,2865,1715,1616,1575,1380,1286,1128,1044 \mathrm{~cm}^{-1}$; HRMS (EI) $m / z[\mathrm{M}]^{\bullet+}$ calcd for $\mathrm{C}_{21} \mathrm{H}_{27} \mathrm{BrO}_{4}$ 422.1093, found 422.1110. Anal. Calcd for $\mathrm{C}_{21} \mathrm{H}_{27} \mathrm{BrO}_{4}$ : C, 59.58; H, 6.43. Found: C, 59.45; H, 6.57.

11-Bromo-2,2,5,7a,10,10,13a-heptamethyl-7a,8,9a,10,11,12,$13,13 a, 13 b, 14-d e c a h y d r o-4 H, 9 H-b e n z o[a][1,3]$ dioxino [5,4-j]xanthen-4-one (44). Bromide 44 (219 mg, $0.446 \mathrm{mmol}, 45 \%, 2: 1 \mathrm{dr})$, prepared from resorcylate $14(413 \mathrm{mg}, 1.00 \mathrm{mmol})$, was obtained as a white solid containing a minor amount of a diastereoisomer: $R_{f} 0.24$ (pentane/ $\mathrm{Et}_{2} \mathrm{O}$ 9:1); mp 234.0-237.4 ${ }^{\circ} \mathrm{C}$; ${ }^{1} \mathrm{H}$ NMR (400 MHz, $\left.\mathrm{CDCl}_{3}\right) \delta 6.33(\mathrm{~s}, 1 \mathrm{H}), 4.00(\mathrm{dd}, J=12.6,4.7 \mathrm{~Hz}, 1 \mathrm{H}), 2.57(\mathrm{~s}, 3 \mathrm{H})$, $2.51(\mathrm{dd}, J=16.8,5.1 \mathrm{~Hz}, 1 \mathrm{H}), 2.37-2.13(\mathrm{~m}, 3 \mathrm{H}), 2.13-2.07(\mathrm{~m}$, $1 \mathrm{H}), 1.91-1.84(\mathrm{~m}, 1 \mathrm{H}), 1.84-1.78(\mathrm{~m}, 1 \mathrm{H}), 1.72(\mathrm{~s}, 3 \mathrm{H}), 1.71-$ $1.69(\mathrm{~m}, 1 \mathrm{H}), 1.69(\mathrm{~s}, 3 \mathrm{H}), 1.59-1.46(\mathrm{~m}, 2 \mathrm{H}), 1.20(\mathrm{~s}, 3 \mathrm{H}), 1.20-$ $1.11(\mathrm{~m}, 2 \mathrm{H}), 1.12(\mathrm{~s}, 3 \mathrm{H}), 0.99(\mathrm{~s}, 3 \mathrm{H}), 0.97(\mathrm{~s}, 3 \mathrm{H}) ;{ }^{13} \mathrm{C}\left\{{ }^{1} \mathrm{H}\right\} \mathrm{NMR}$ $\left(101 \mathrm{MHz}, \mathrm{CDCl}_{3}\right) \delta 160.8,158.5,156.1,142.2,114.3,107.8,104.8$, 104.1, 77.8, 68.4, 56.3, 51.1, 40.7, 40.5, 39.6, 37.0, 30.7, 30.6, 26.1, $25.5,22.0,21.1,20.6,18.3,16.5,14.9$; IR $\nu_{\max }$ (neat) 2949, 1718, 1613, 1575, 1376, 1288, 1131, 1042, 902, 844, $693 \mathrm{~cm}^{-1}$; HRMS (ESI) $\mathrm{m} / z[\mathrm{M}+\mathrm{H}]^{+}$calcd for $\mathrm{C}_{26} \mathrm{H}_{36} \mathrm{BrO}_{4}$ 491.1797, found 491.1807. Anal. Calcd for $\mathrm{C}_{26} \mathrm{H}_{35} \mathrm{BrO}_{4}$ : $\mathrm{C}, 63.54 ; \mathrm{H}, 7.18$. Found: $\mathrm{C}$, 63.49; H, 7.07.

General Procedure for IDSI (47)-Induced Halocyclization. IDSI (47) (884 mg, $1.10 \mathrm{mmol})$ was added with stirring to resorcylate 24 or $14(1.00 \mathrm{mmol})$ in $\mathrm{MeNO}_{2}(50 \mathrm{~mL})$ at $-25^{\circ} \mathrm{C}$. After $10 \mathrm{~min}$, saturated aqueous $\mathrm{NaHCO}_{3}(15 \mathrm{~mL})$ and aqueous $\mathrm{Na}_{2} \mathrm{SO}_{3}(0.5 \mathrm{M} ; 5$ $\mathrm{mL}$ ) were added, and stirring was continued for $15 \mathrm{~min}$. The two phases were separated, and the aqueous layer was extracted with $\mathrm{CH}_{2} \mathrm{Cl}_{2}(3 \times 10 \mathrm{~mL})$. The combined organic layers were dried $\left(\mathrm{MgSO}_{4}\right)$, filtered, concentrated, and chromatographed (pentane/ $\left.\mathrm{Et}_{2} \mathrm{O} 9: 1\right)$ to give the iodo-meroterpenoid 43 or $\mathbf{4 5}$.

10-Iodo-2,2,5,7a, 11,11-hexamethyl-7a,8,10,11,11a,12-hexahydro-4H,9H-[1,3]dioxino[4,5-a]xanthen-4-one (43). Iodide 43 (415 $\mathrm{mg}, 0.882 \mathrm{mmol}, 88 \%)$, prepared from resorcylate $24(344 \mathrm{mg}, 1.00$ mmol), was obtained as a white solid: $R_{f} 0.29$ (pentane/ $\mathrm{Et}_{2} \mathrm{O} 9: 1$ ); mp 221.6-222.4 ${ }^{\circ} \mathrm{C} ;{ }^{1} \mathrm{H}$ NMR $\left(400 \mathrm{MHz},\left(\mathrm{CD}_{3}\right)_{2} \mathrm{CO}\right) \delta 6.36(\mathrm{~s}$, $1 \mathrm{H}), 4.46(\mathrm{dd}, J=11.9,4.8 \mathrm{~Hz}, 1 \mathrm{H}), 2.82(\mathrm{dd}, J=23.3,6.7 \mathrm{~Hz}, 1 \mathrm{H})$, $2.52(\mathrm{~s}, 3 \mathrm{H}), 2.50-2.44(\mathrm{~m}, 1 \mathrm{H}), 2.46-2.30(\mathrm{~m}, 2 \mathrm{H}), 1.91(\mathrm{dd}, J=$ $13.2,5.0 \mathrm{~Hz}, 1 \mathrm{H}), 1.87-1.82(\mathrm{~m}, 2 \mathrm{H}), 1.70(\mathrm{~s}, 3 \mathrm{H}), 1.67(\mathrm{~s}, 3 \mathrm{H})$, $1.28(\mathrm{~s}, 3 \mathrm{H}), 1.16(\mathrm{~s}, 3 \mathrm{H}), 1.09(\mathrm{~s}, 3 \mathrm{H}) ;{ }^{13} \mathrm{C}\left\{{ }^{1} \mathrm{H}\right\}$ NMR $(100 \mathrm{MHz}$, $\left.\left(\mathrm{CD}_{3}\right)_{2} \mathrm{CO}\right) \delta 160.5,158.9,156.7,142.4,114.9,109.3,105.6,105.2$, 78.2, 50.1, 46.2, 42.5, 39.6, 34.9, 32.3, 26.1, 25.4, 21.9, 20.3, 20.0, 
19.9; IR $\nu_{\max }$ (neat) 2970, 1715, 1616, 1575, 1379, 1285, 1127, 1044, $902 \mathrm{~cm}^{-1}$; HRMS (EI) $\mathrm{m} / z[\mathrm{M}]^{\bullet+}$ Calcd for $\mathrm{C}_{21} \mathrm{H}_{27} \mathrm{IO}_{4} 470.0954$, found 470.0947. Anal. Calcd for $\mathrm{C}_{21} \mathrm{H}_{27} \mathrm{IO}_{4}$ : C, 53.63; $\mathrm{H}, 5.79$. Found: C, 53.55; H, 5.86 .

11-lodo-2,2,5,7a,10,10,13a-heptamethyl-7a, 8,9a, 10,11,12, $13,13 a, 13 b, 14-d e c a h y d r o-4 H, 9 H$-benzo[a][1,3]dioxino $[5,4-j]-$ xanthen-4-one (45). Iodide 45 (293 mg, $0.544 \mathrm{mmol}, 54 \%, 2: 1 \mathrm{dr}$ ), prepared from resorcylate $14(413 \mathrm{mg}, 1.00 \mathrm{mmol})$, was obtained as a white solid containing a minor diastereoisomer: $R_{f} 0.24$ (pentane/ $\mathrm{Et}_{2} \mathrm{O}$ 9:1); mp 189.5-192.0 ${ }^{\circ} \mathrm{C}$; ${ }^{1} \mathrm{H}$ NMR (400 MHz, $\left.\mathrm{CDCl}_{3}\right) \delta 6.33$ $(\mathrm{s}, 1 \mathrm{H}), 4.24(\mathrm{dd}, J=13.0,3.2 \mathrm{~Hz}, 1 \mathrm{H}), 2.57(\mathrm{~s}, 3 \mathrm{H}), 2.50(\mathrm{dd}, J=$ 16.4, $5.0 \mathrm{~Hz}, 1 \mathrm{H}), 2.46-2.29(\mathrm{~m}, 2 \mathrm{H}), 2.24(\mathrm{dd}, J=16.8,13.1 \mathrm{~Hz}$, $1 \mathrm{H}), 2.10-2.03(\mathrm{~m}, 1 \mathrm{H}), 1.95-1.87(\mathrm{~m}, 1 \mathrm{H}), 1.72(\mathrm{~s}, 3 \mathrm{H}), 1.69(\mathrm{~s}$, $3 \mathrm{H}), 1.68-1.55(\mathrm{~m}, 2 \mathrm{H}), 1.55-1.51(\mathrm{~m}, 1 \mathrm{H}), 1.51-1.45(\mathrm{~m}, 1 \mathrm{H})$, $1.26-1.20(\mathrm{~m}, 1 \mathrm{H}), 1.20(\mathrm{~s}, 3 \mathrm{H}), 1.20-1.10(\mathrm{~m}, 1 \mathrm{H}), 1.10(\mathrm{~s}, 3 \mathrm{H})$, $1.01(\mathrm{~s}, 3 \mathrm{H}), 0.98(\mathrm{~s}, 3 \mathrm{H}) ;{ }^{13} \mathrm{C}\left\{{ }^{1} \mathrm{H}\right\} \mathrm{NMR}\left(101 \mathrm{MHz}, \mathrm{CDCl}_{3}\right) \delta$ $160.8,158.5,156.1,142.2,114.3,107.8,104.8,104.1,77.3,54.9,52.9$, 51.2, 42.2, 40.8, 39.3, 37.3, 33.6, 33.2, 26.1, 25.5, 22.2, 22.0, 21.1, 20.6, 16.5, 14.9; IR $\nu_{\max }$ (neat) 2946, 1726, 1615, 1576, 1371, 1285, 1124, 1038, $902 \mathrm{~cm}^{-1}$; HRMS (ESI) $\mathrm{m} / z[\mathrm{M}+\mathrm{H}]^{+}$calcd for $\mathrm{C}_{26} \mathrm{H}_{36} \mathrm{IO}_{4} 539.1658$, found 539.1664 .

2,2,5,7a, 11,11-Hexamethyl-7a,8,10,11,11a,12-hexahydro-4H,9H$[1,3]$ dioxino $[4,5-a]$ xanthen-4-one (48). $\mathrm{BF}_{3} \cdot \mathrm{OEt}_{2}(0.31 \mathrm{~mL}, 2.50$ $\mathrm{mmol})$ was added dropwise with stirring to resorcylate $24(172 \mathrm{mg}$, $0.50 \mathrm{mmol})$ in $\mathrm{CH}_{2} \mathrm{Cl}_{2}(50 \mathrm{~mL})$ at $-78^{\circ} \mathrm{C}$. The resulting mixture was warmed to $25{ }^{\circ} \mathrm{C}$ and further stirred for $1.5 \mathrm{~h}$, when saturated aqueous $\mathrm{NaHCO}_{3}(25 \mathrm{~mL})$ and $\mathrm{H}_{2} \mathrm{O}(25 \mathrm{~mL})$ were added and the phases were separated. The aqueous layer was extracted with $\mathrm{CH}_{2} \mathrm{Cl}_{2}$ $(3 \times 25 \mathrm{~mL})$, and the combined organic layers were dried $\left(\mathrm{MgSO}_{4}\right)$, filtered, concentrated, and chromatographed (pentane/ $\mathrm{Et}_{2} \mathrm{O} 9: 1$ ) to provide meroterpenoid 48 (154 $\mathrm{mg}, 0.447 \mathrm{mmol}, 89 \%, 3: 1 \mathrm{dr}$ ) as a white solid containing a minor diastereoisomer. Recrystallization from $n$-hexane provided the pure trans-fused ring product: $R_{f} 0.48$ (pentane/EtOAc 19:1); mp 139.5-141.3 ${ }^{\circ} \mathrm{C}$; ${ }^{1} \mathrm{H}$ NMR (400 MHz, $\left.\mathrm{CDCl}_{3}\right) \delta 6.34(\mathrm{~s}, 1 \mathrm{H}), 2.62(\mathrm{dd}, J=16.8,4.8 \mathrm{~Hz}, 1 \mathrm{H}), 2.57(\mathrm{~s}, 3 \mathrm{H})$, $2.22(\mathrm{dd}, J=16.8,13.3 \mathrm{~Hz}, 1 \mathrm{H}), 2.01-1.94(\mathrm{~m}, 1 \mathrm{H}), 1.74(\mathrm{~s}, 3 \mathrm{H})$, $1.70(\mathrm{~s}, 3 \mathrm{H}), 1.68-1.64(\mathrm{~m}, 1 \mathrm{H}), 1.60(\mathrm{dd}, J=13.4,4.8 \mathrm{~Hz}, 1 \mathrm{H})$, $1.60-1.55(\mathrm{~m}, 2 \mathrm{H}), 1.54-1.47(\mathrm{~m}, 1 \mathrm{H}), 1.37-1.28(\mathrm{~m}, 1 \mathrm{H}), 1.21(\mathrm{~s}$, $3 \mathrm{H}), 1.03(\mathrm{~s}, 3 \mathrm{H}), 0.93(\mathrm{~s}, 3 \mathrm{H}) ;{ }^{13} \mathrm{C}\left\{{ }^{1} \mathrm{H}\right\} \operatorname{NMR}\left(100 \mathrm{MHz} \mathrm{CDCl}_{3}\right) \delta$ $160.9,158.8,156.0,142.1,114.4,108.6,104.8,104.0,78.7,47.4,41.4$, $39.7,33.5,32.1,26.2,25.5,22.0,20.6,19.8,19.7,17.3$; IR $\nu_{\max }$ (neat) 2969, 2901, 2921, 1720, 1618, 1576, 1390, 1286, $1043 \mathrm{~cm}^{-1}$; HRMS (ESI) $m / z[\mathrm{M}]^{\bullet+}$ calcd for $\mathrm{C}_{21} \mathrm{H}_{28} \mathrm{O}_{4} 344.1988$, found 344.1990. Anal. Calcd for $\mathrm{C}_{21} \mathrm{H}_{28} \mathrm{O}_{4}$ : C, 73.32; H, 8.19. Found: C, 73.17; H, 8.22.

8-Hydroxy-1,1,4a,6-tetramethyl-2,3,4,4a,9,9a-hexahydro- $1 \mathrm{H}$ xanthene-7-carboxylic Acid (49). $\mathrm{H}_{2} \mathrm{O}(8 \mu \mathrm{L}, 0.4 \mathrm{mmol})$ was added with stirring to a suspension of $\mathrm{KO}^{t} \mathrm{Bu}(180 \mathrm{mg}, 1.60 \mathrm{mmol})$ in $\mathrm{Et}_{2} \mathrm{O}$ $(3 \mathrm{~mL})$ at $0{ }^{\circ} \mathrm{C}$. After $5 \mathrm{~min}$, meroterpenoid $48(69 \mathrm{mg}, 0.200 \mathrm{mmol})$ was added, and the resulting mixture was further stirred at $25^{\circ} \mathrm{C}$ for 2 h. The $\mathrm{pH}$ was adjusted to $\sim 2$ with aqueous $\mathrm{HCl}(1 \mathrm{M})$, the two phases were separated, and the aqueous layer was extracted with $\mathrm{Et}_{2} \mathrm{O}$ $(3 \times 10 \mathrm{~mL})$. The combined organic layers were dried $\left(\mathrm{MgSO}_{4}\right)$, filtered, concentrated, and chromatographed (pentane/EtOAc/AcOH 9:1:0.01) to give the carboxylic acid $49(42 \mathrm{mg}, 0.138 \mathrm{mmol}, 69 \%)$ as a white solid: $R_{f} 0.30$ (pentane/EtOAc/AcOH 9:1:0.01); mp 176.7$178.6{ }^{\circ} \mathrm{C} ;{ }^{1} \mathrm{H}$ NMR $\left(400 \mathrm{MHz}, \mathrm{CDCl}_{3}\right) \delta 11.84(\mathrm{~s}, 1 \mathrm{H}), 11.17(\mathrm{~s}$, $1 \mathrm{H}), 6.22(\mathrm{~s}, 1 \mathrm{H}), 2.77(\mathrm{dd}, J=17.0,4.8 \mathrm{~Hz}, 1 \mathrm{H}), 2.52(\mathrm{~s}, 3 \mathrm{H}), 2.28$ $(\mathrm{dd}, J=16.9,13.4 \mathrm{~Hz}, 1 \mathrm{H}), 2.01-1.94(\mathrm{~m}, 1 \mathrm{H}), 1.72-1.64(\mathrm{~m}, 1 \mathrm{H})$, $1.64-1.55(\mathrm{~m}, 3 \mathrm{H}), 1.55-1.45(\mathrm{~m}, 1 \mathrm{H}), 1.40-1.27(\mathrm{~m}, 1 \mathrm{H}), 1.22(\mathrm{~s}$, $3 \mathrm{H}), 1.04(\mathrm{~s}, 3 \mathrm{H}), 0.94(\mathrm{~s}, 3 \mathrm{H}) ;{ }^{13} \mathrm{C}\left\{{ }^{1} \mathrm{H}\right\} \operatorname{NMR}\left(100 \mathrm{MHz}, \mathrm{CDCl}_{3}\right) \delta$ 175.5, 163.7, 158.9, 141.4, 112.7, 108.4, 102.4, 78.7, 47.6, 41.5, 39.7, 33.6, 32.1, 24.1, 20.6, 19.8, 19.7, 17.6; IR $\nu_{\max }$ (neat) 2694, 2919, 2865, 1619, 1578, 1454,1268, 1151, $1100 \mathrm{~cm}^{-1}$; HRMS (EI) $\mathrm{m} / z$ $[\mathrm{M}]^{\bullet+}$ Calcd for $\mathrm{C}_{18} \mathrm{H}_{24} \mathrm{O}_{4}$ 304.1675, found 304.1679. Anal. Calcd for $\mathrm{C}_{18} \mathrm{H}_{24} \mathrm{O}_{4}$ : C, 71.03; H, 7.95. Found: C, 71.17; H, 8.11.

1,1,4a,6-Tetramethyl-2,3,4,4a,9,9a-hexahydro-1H-xanthen-8-ol (50). Aqueous $\mathrm{KOH}(5 \mathrm{M} ; 1 \mathrm{~mL})$ was added with stirring to meroterpenoid $48(69 \mathrm{mg}, 0.200 \mathrm{mmol})$ in 1,4-dioxane $(2 \mathrm{~mL})$, and the resulting mixture was heated at $110{ }^{\circ} \mathrm{C}$ for $23 \mathrm{~h}$. After the reaction mixture was cooled to $25^{\circ} \mathrm{C}$, the $\mathrm{pH}$ was adjusted to $\sim 2$ with aqueous
$\mathrm{HCl}(4 \mathrm{M})$. The two phases were separated, and the aqueous layer was extracted with $\mathrm{Et}_{2} \mathrm{O}(3 \times 10 \mathrm{~mL})$. The combined organic layers were dried $\left(\mathrm{MgSO}_{4}\right)$, filtered, concentrated, and chromatographed (pentane/EtOAc 15:1) to give the phenol 50 (40 mg, $0.194 \mathrm{mmol}$, 97\%) as a white foam: $R_{f} 0.33$ (pentane/EtOAc 15:1); ${ }^{1} \mathrm{H}$ NMR (400 $\left.\mathrm{MHz},\left(\mathrm{CD}_{3}\right)_{2} \mathrm{CO}\right) \delta 8.00(\mathrm{~s}, 1 \mathrm{H}), 6.21(\mathrm{~s}, 1 \mathrm{H}), 6.05(\mathrm{~s}, 1 \mathrm{H}), 2.71$ (dd, $J=16.7,5.0 \mathrm{~Hz}, 1 \mathrm{H}), 2.26(\mathrm{dd}, J=16.6,13.5 \mathrm{~Hz}, 1 \mathrm{H}), 2.12(\mathrm{~s}$, $3 \mathrm{H}), 1.92-1.81(\mathrm{~m}, 1 \mathrm{H}), 1.68-1.53(\mathrm{~m}, 4 \mathrm{H}), 1.52-1.44(\mathrm{~m}, 1 \mathrm{H})$, $1.40-1.27(\mathrm{~m}, 1 \mathrm{H}), 1.17(\mathrm{~s}, 3 \mathrm{H}), 1.01(\mathrm{~s}, 3 \mathrm{H}), 0.94(\mathrm{~s}, 3 \mathrm{H}) ;{ }^{13} \mathrm{C}\left\{{ }^{1} \mathrm{H}\right\}$ NMR $\left(100 \mathrm{MHz},\left(\mathrm{CD}_{3}\right)_{2} \mathrm{CO}\right) \delta 156.2,155.0,137.2,109.7,107.7$, 107.6, 77.0, 48.8, 42.3, 40.8, 34.0, 21.3, 20.9, 20.4, 20.0, 18.6; IR $\nu_{\max }$ (neat) $3398,2935,2866,1627,1587,1516,1457,1101,1063,1040$ $\mathrm{cm}^{-1}$; HRMS (EI) $\mathrm{m} / z[\mathrm{M}]^{\bullet+}$ calcd for $\mathrm{C}_{17} \mathrm{H}_{24} \mathrm{O}_{2} 260.1776$, found 260.1786 .

7-(Hydroxymethyl)-1, 1,4a,6-tetramethyl-2,3,4,4a,9,9a-hexahydro-1H-xanthen-8-ol (51). $\mathrm{LiAlH}_{4}$ in THF $(1 \mathrm{M} ; 0.8 \mathrm{~mL}, 0.800$ $\mathrm{mmol})$ was added with stirring to meroterpenoid $48(69 \mathrm{mg}, 0.200$ $\mathrm{mmol})$ in THF $(4 \mathrm{~mL})$ at $0{ }^{\circ} \mathrm{C}$. After $1 \mathrm{~h}, \mathrm{H}_{2} \mathrm{O}(65 \mu \mathrm{L})$ and $\mathrm{NaOH}$ $(1 \mathrm{M} ; 150 \mu \mathrm{L})$ were added dropwise sequentially, and the mixture was further stirred for $30 \mathrm{~min}$. Solid $\mathrm{NH}_{4} \mathrm{Cl}(100 \mathrm{mg})$ was added, and the solids were filtered and eluted with $\mathrm{Et}_{2} \mathrm{O}(5 \mathrm{~mL})$. The filtrate was concentrated and chromatographed (pentane/EtOAc 4:1) to afford diol 51 (55 mg, $0.189 \mathrm{mmol}, 95 \%)$ as white foam: $R_{f} 0.22$ (pentane/ EtOAc 9:1); ${ }^{1} \mathrm{H}$ NMR (400 MHz, $\left.\mathrm{CDCl}_{3}\right) \delta 7.88(\mathrm{~s}, 1 \mathrm{H}), 6.19(\mathrm{~s}$, $1 \mathrm{H}), 4.88(\mathrm{~s}, 2 \mathrm{H}), 2.75(\mathrm{dd}, J=16.6,5.0 \mathrm{~Hz}, 1 \mathrm{H}), 2.37-2.27(\mathrm{~m}$, $1 \mathrm{H}), 2.17(\mathrm{~s}, 3 \mathrm{H}), 1.99-1.90(\mathrm{~m}, 1 \mathrm{H}), 1.69-1.54(\mathrm{~m}, 4 \mathrm{H}), 1.52-$ $1.40(\mathrm{~m}, 1 \mathrm{H}), 1.38-1.25(\mathrm{~m}, 1 \mathrm{H}), 1.20(\mathrm{~s}, 3 \mathrm{H}), 1.02(\mathrm{~s}, 3 \mathrm{H}), 0.92(\mathrm{~s}$, $3 \mathrm{H}) ;{ }^{13} \mathrm{C}\left\{{ }^{1} \mathrm{H}\right\}$ NMR $\left(100 \mathrm{MHz}, \mathrm{CDCl}_{3}\right) \delta 155.0,153.4,133.6,113.6$, $110.2,109.0,77.1,60.8,47.8,41.6,39.9,33.5,32.1,20.6,19.8,19.6$, 19.2, 17.7; IR $\nu_{\max }$ (neat) 3344, 2934, 2865, 1627, 1583, $1102 \mathrm{~cm}^{-1}$; HRMS (ESI) $m / z[M-H]^{-}$calcd for $\mathrm{C}_{18} \mathrm{H}_{25} \mathrm{O}_{3}$ 289.1804, found 289.1807. Anal. Calcd for $\mathrm{C}_{18} \mathrm{H}_{26} \mathrm{O}_{3}$ : C, 74.45; H, 9.02. Found: C, 74.59; H, 9.13.

8-Hydroxy-N-methoxy- $N, 1,1,4 a, 6$-pentamethyl-2,3,4,4a,9,9ahexahydro- $1 \mathrm{H}$-xanthene-7-carboxamide (52). $\mathrm{MeNH}(\mathrm{OMe}) \cdot \mathrm{HCl}$ (59 $\mathrm{mg}, 0.605 \mathrm{mmol}$ ) was added dropwise with stirring to meroterpenoid $48(69 \mathrm{mg}, 0.200 \mathrm{mmol})$ in THF $(4 \mathrm{~mL})$ and cooled to $0{ }^{\circ} \mathrm{C}$, followed by dropwise addition of ${ }^{i} \mathrm{PrMgCl}$ in THF $(2 \mathrm{M}$; 0.6 $\mathrm{mL}, 1.20 \mathrm{mmol})$. The resulting mixture was stirred at $0{ }^{\circ} \mathrm{C}$ for $3 \mathrm{~h}$, when the reaction was quenched with saturated $\mathrm{NH}_{4} \mathrm{Cl}(2 \mathrm{~mL})$ and the mixture was acidified to $\mathrm{pH} \sim 1$ with aqueous $\mathrm{HCl}(1 \mathrm{M})$. The two phases were separated, and the aqueous layer was extracted with EtOAc $(3 \times 5 \mathrm{~mL})$. The combined organic layers were dried $\left(\mathrm{MgSO}_{4}\right)$, filtered, concentrated, and chromatographed (pentane/ EtOAc 7:3) to give the amide $\mathbf{5 2}(67 \mathrm{mg}, 0.193 \mathrm{mmol}, 96 \%)$ as a white solid: $R_{f} 0.43$ (pentane/EtOAc 7:3); mp $178.9-182.7{ }^{\circ} \mathrm{C} ;{ }^{1} \mathrm{H}$ $\operatorname{NMR}\left(400 \mathrm{MHz}, \mathrm{CDCl}_{3}\right) \delta 6.20(\mathrm{~s}, 1 \mathrm{H}), 3.57(\mathrm{~s}, 3 \mathrm{H}), 3.33(\mathrm{~s}, 3 \mathrm{H})$, $2.74(\mathrm{dd}, J=16.7,5.0 \mathrm{~Hz}, 1 \mathrm{H}), 2.28(\mathrm{dd}, J=16.7,13.4 \mathrm{~Hz}, 1 \mathrm{H}), 2.23$ $(\mathrm{s}, 3 \mathrm{H}), 1.98-1.93(\mathrm{~m}, 1 \mathrm{H}), 1.71-1.64(\mathrm{~m}, 1 \mathrm{H}), 1.61(\mathrm{dd}, J=13.2$, $4.9 \mathrm{~Hz}, 1 \mathrm{H}), 1.59-1.54(\mathrm{~m}, 2 \mathrm{H}), 1.54-1.45(\mathrm{~m}, 1 \mathrm{H}), 1.37-1.24(\mathrm{~m}$, $1 \mathrm{H}), 1.20(\mathrm{~s}, 3 \mathrm{H}), 1.02(\mathrm{~s}, 3 \mathrm{H}), 0.92(\mathrm{~s}, 3 \mathrm{H}) ;{ }^{13} \mathrm{C}\left\{{ }^{1} \mathrm{H}\right\}$ NMR $(100$ $\left.\mathrm{MHz}, \mathrm{CDCl}_{3}\right) \delta 169.9,155.5,154.5,135.3,111.1,110.7,108.5,77.7$, 61.3, 47.7, 41.6, 39.9, 34.2, 33.5, 32.1, 20.6, 19.9, 19.8, 19.7, 17.8; IR $\nu_{\max }$ (neat) 3110, 2933,1616,1578,1456,1389, 1120, $732 \mathrm{~cm}^{-1}$; HRMS (ESI) $m / z[\mathrm{M}+\mathrm{H}]^{+}$calcd for $\mathrm{C}_{20} \mathrm{H}_{30} \mathrm{NO}_{4} 348.2175$, found 348.2188. Anal. Calcd for $\mathrm{C}_{20} \mathrm{H}_{29} \mathrm{NO}_{4}$ : C, 69.14; $\mathrm{H}, 8.41 ; \mathrm{N}, 4.03$. Found: C, 69.11; H, 8.49; N, 3.97.

\section{ASSOCIATED CONTENT}

\section{Supporting Information}

The Supporting Information is available free of charge on the ACS Publications website at DOI: 10.1021/acs.joc.8b02095.

${ }^{1} \mathrm{H}$ and ${ }^{13} \mathrm{C}$ NMR spectra for (+)-hongoquercin A (1), (+)-hongoquercin B (2), and compounds 14, 20, 21, 24, $27,28,30,31,33-35,37,39-41,42-45$, and 48-52 (PDF)

X-ray structural data for compounds $1,34,41-45$, and 48 (PDF) 
X-ray structural data for compounds $1,34,41-45$, and 48 (CIF)

\section{AUTHOR INFORMATION}

\section{Corresponding Authors}

*E-mail: p.parsons@imperial.ac.uk.

*E-mail: agm.barrett@imperial.ac.uk.

ORCID $\odot$

Anthony G. M. Barrett: 0000-0002-8485-215X

Notes

The authors declare no competing financial interest.

\section{ACKNOWLEDGMENTS}

We thank GlaxoSmithKline for the endowment (to A.G.M.B) as well as Drs. Alfred and Isabel Bader for their additional support.

\section{REFERENCES}

(1) (a) Roll, D. M.; Manning, J. K.; Carter, G. T. Hongoquercins A and B, New Sesquiterpenoid Antibiotics: Isolation, Structure Elucidation, and Antibacterial Activity. J. Antibiot. 1998, 51 (7), 635-639. (b) Abbanat, D. A.; Singh, M. P.; Greenstein, M. Hongoquercins, New Antibacterial Agents from the Fungus LL23G227: Fermentation and Biological Activity. J. Antibiot. 1998, 51 (8), 708-714.

(2) (a) Hirai, K.; Suzuki, K. T.; Nozoe, S. The Structure and the Chemistry of Siccanin and Related Compounds. Tetrahedron 1971, 27 (24), 6057-6061. (b) Horak, R. M.; Steyn, P. S.; Vleggaar, R.; Rabie, C. J. Metabolites of Aspergillus Ustus. Part 3. Structure Elucidation of Austalides G-L. J. Chem. Soc., Perkin Trans. 1 1985, 47, 363-367.

(3) (a) Tsujimori, H.; Bando, M.; Mori, K. Synthesis and Absolute Configuration of Hongoquercin A, an Antibacterial SesquiterpeneSubstituted Orsellinic Acid Isolated as a Fungal Metabolite. Eur. J. Org. Chem. 2000, 2000 (2), 297-302. (b) Tsujimori, H.; Mori, K. Synthesis and Absolute Configuration of Hongoquercin B, a Sesquiterpene-Substituted Orsellinic Acid Isolated as a Fungal Metabolite. Biosci., Biotechnol., Biochem. 2000, 64 (7), 1410-1415. (c) Kurdyumov, A. V.; Hsung, R. P. An Unusual Cationic $[2+2]$ Cycloaddition in a Divergent Total Synthesis of Hongoquercin A and Rhododaurichromanic Acid A. J. Am. Chem. Soc. 2006, 128 (19), 6272-6273. (d) Rosen, B. R.; Simke, L. R.; Thuy-Boun, P. S.; Dixon, D. D.; Yu, J.-Q.; Baran, P. S. C-H. Functionalization Logic Enables Synthesis of (+)-Hongoquercin A and Related Compounds. Angew. Chem., Int. Ed. 2013, 52 (28), 7317-7320. (e) Fernández, A.; Alvarez, E.; Alvarez-Manzaneda, R.; Chahboun, R.; Alvarez-Manzaneda, E. A Short Synthetic Route towards Merosesquiterpenes with a Benzoxanthene Skeleton. Chem. Commun. 2014, 50 (86), 13100-13102. (f) Dethe, D. H.; Murhade, G. M.; Dherange, B. D.; Sau, S. K. Enantiospecific Syntheses of Hongoquercins A and B and Chromazonarol. Eur. J. Org. Chem. 2017, 2017 (7), 1143-1150. (g) Yang, Z.; Li, S.; Luo, S. Total Synthesis of ( \pm )-Hongoquercin A via Visible-Light-Mediated Organocatalytic Polyene Cyclization. Huaxue Xuebao 2017, 75 (4), 351-354.

(4) Danheiser, R. L.; Gee, S. K. A Regiocontrolled Annulation Approach to Highly Substituted Aromatic Compounds. J. Org. Chem. 1984, 49 (9), 1672-1674.

(5) Harris, T. M.; Harris, C. M. Synthesis of Polyketide-Type Aromatic Natural Products by Biogenetically Modeled Routes. Tetrahedron 1977, 33 (17), 2159-2185.

(6) Hyatt, J. A.; Feldman, P. L.; Clemens, R. J. Ketenes. 20. Thermal Decomposition of 2,2,6-Trimethyl-4H-1,3-Dioxin-4-One and 1Ethoxybutyn-3-One. Acetylketene. J. Org. Chem. 1984, 49 (26), $5105-5108$

(7) Cookson, R.; Barrett, T. N.; Barrett, A. G. M. $\beta$-Keto-Dioxinones and $\beta, \delta$-Diketo-Dioxinones in Biomimetic Resorcylate Total Synthesis. Acc. Chem. Res. 2015, 48 (3), 628-642.
(8) Calo, F.; Richardson, J.; Barrett, A. G. M. Total Synthesis of Aigialomycin D Using a One-Pot Ketene Generation-TrappingAromatization Sequence. Org. Lett. 2009, 11 (21), 4910-4913.

(9) (a) Anderson, K.; Calo, F.; Pfaffeneder, T.; White, A. J. P.; Barrett, A. G. M. Biomimetic Total Synthesis of Angelicoin A and B via a Palladium-Catalyzed Decarboxylative Prenylation-Aromatization Sequence. Org. Lett. 2011, 13 (21), 5748-5750. (b) Laclef, S.; Anderson, K.; White, A. J. P.; Barrett, A. G. M. Total Synthesis of Amorfrutin A via a Palladium-Catalyzed Migratory Prenylationaromatization Sequence. Tetrahedron Lett. 2012, 53 (2), 225-227. (c) Cordes, J.; Calo, F.; Anderson, K.; Pfaffeneder, T.; Laclef, S.; White, A. J. P.; Barrett, A. G. M. Total Syntheses of Angelicoin A, Hericenone J, and Hericenol A via Migratory Prenyl- and Geranylation-Aromatization Sequences. J. Org. Chem. 2012, 77 (1), 652-657. (d) Brookes, P. a.; Cordes, J.; White, A. J. P.; Barrett, A. G. M. Total Synthesis of Mycophenolic Acid by a Palladium-Catalyzed Decarboxylative Allylation and Biomimetic Aromatization Sequence. Eur. J. Org. Chem. 2013, 2013 (32), 7313-7319. (e) Barrett, T. N.; Barrett, A. G. M. Cascade Polyketide and Polyene Cyclizations: Biomimetic Total Synthesis of Hongoquercin B. J. Am. Chem. Soc. 2014, 136 (49), 17013-17015.

(10) (a) Elliott, D. C.; Ma, T.-K.; Selmani, A.; Cookson, R.; Parsons, P. J.; Barrett, A. G. M. Sequential Ketene Generation from Dioxane4,6-Dione-Keto-Dioxinones for the Synthesis of Terpenoid Resorcylates. Org. Lett. 2016, 18 (8), 1800-1803. (b) Ma, T.-K.; White, A. J. P.; Barrett, A. G. M. Meroterpenoid Total Synthesis: Conversion of Geraniol and Farnesol into Amorphastilbol, Grifolin and Grifolic Acid by Dioxinone- $\beta$-Keto-Acylation, Palladium Catalyzed Decarboxylative Allylic Rearrangement and Aromatization. Tetrahedron Lett. 2017, 58 (28), 2765-2767.

(11) Surendra, K.; Corey, E. J. Highly Enantioselective ProtonInitiated Polycyclization of Polyenes. J. Am. Chem. Soc. 2012, 134 (29), 11992-11994.

(12) Ishibashi, H.; Ishihara, K.; Yamamoto, H. A New Artificial Cyclase for Polyprenoids: Enantioselective Total Synthesis of (-)-Chromazonarol, (+)-8-epi-Puupehedione, and (-)-11'-Deoxytaondiol Methyl Ether. J. Am. Chem. Soc. 2004, 126 (36), 1112211123.

(13) Gassman, P. G.; Schenk, W. N. A General Procedure for the Base-Promoted Hydrolysis of Hindered Esters at Ambient Temperatures. J. Org. Chem. 1977, 42 (5), 918-920.

(14) Lattanzi, A.; Scettri, A. VO(Acac) $)_{2} /$ TBHP Catalyzed Epoxidation of 2-(2-Alkenyl)phenols. Highly Regio- and Diastereoselective Oxidative Cyclization to 2,3-Dihydro-Benzofuranols and 3-Chromanols. Synlett 2002, 2002 (6), 942-946.

(15) Corey, E. J.; Zhang, J. Highly Effective Transition Structure Designed Catalyst for the Enantio- and Position- Selective Dihydroxylation of Polyisoprenoids. Org. Lett. 2001, 3 (20), 32113214.

(16) Garro-Helion, F.; Merzouk, A.; Guibe, F. Mild and Selective Palladium(0)-Catalyzed Deallylation of Allylic Amines. Allylamine and Diallylamine as Very Convenient Ammonia Equivalents for the Synthesis of Primary Amines. J. Org. Chem. 1993, 58 (22), 61096113.

(17) Sen, S. E.; Roach, S. L.; Smith, S. M.; Zhang, Y. Z. Ferric Chloride, an Efficient Promoter of Cationic Polyene Cyclizations. Tetrahedron Lett. 1998, 39 (23), 3969-3972.

(18) (a) Wang, Z.-X.; Tu, Y.; Frohn, M.; Zhang, J.-R.; Shi, Y. An Efficient Catalytic Asymmetric Epoxidation Method. J. Am. Chem. Soc. 1997, 119 (46), 11224-11235. (b) Zhao, M.-X.; Shi, Y. Practical Synthesis of an L-Fructose-Derived Ketone Catalyst for Asymmetric Epoxidation of Olefins. J. Org. Chem. 2006, 71 (14), 5377-5379.

(19) Snyder, S. A.; Treitler, D. S.; Brucks, A. P. Simple Reagents for Direct Halonium-Induced Polyene Cyclizations. J. Am. Chem. Soc. 2010, 132 (40), 14303-14314.

(20) Bromide 40 and iodide $\mathbf{4 1}$ were isolated as an inseparable 2:1 mixture of diastereoisomers about the highlighted carbon atom favoring the drawn diastereoisomer. 
(21) Meroterpenoid $\mathbf{4 2}$ was isolated as an inseparable 3:1 mixture of diastereoisomers about the highlighted carbon atom favoring the drawn diastereoisomer.

(22) Barrett, T. N.; Patel, B. H.; Barrett, A. G. M. Synthesis of C-5Substituted Resorcylates and Resorcinamides via Formylationaromatization of Functionalized Keto-Dioxinones. Tetrahedron 2014, 70 (38), 6894-6901. 